This peer reviewd manuscript has been accepted for publication in Physics of Fluids

https://doi.org/10.1063/1.5000413

\title{
Viscoelasticity of new generation thermoplastic polyurethane vibration isolators ${ }^{\mathrm{a})}$
}

Marko Bek, ${ }^{\text {b) }}$ Joris Betjes ${ }^{\mathrm{b})}$, Bernd-Steffen von Bernstorff, ${ }^{\mathrm{c})}$ and Igor Emri ${ }^{\mathrm{b})}$

(Dated: 1 December 2017)

This paper presents the analysis of pressure dependence of three thermoplastic polyurethane (TPU) materials on vibration isolation. The three TPU Elastollan ${ }^{\circledR}$ materials are: 1190A, 1175A and 1195D. Aim of this investigation was to analyze how much the performance of isolation can be enhanced using patented Dissipative bulk and granular systems technology. The technology uses granular polymeric materials to enhance materials properties (without changing its chemical or molecular composition) by exposing them to 'self-pressurization', which shifts material energy absorption maxima towards lower frequencies, to match the excitation frequency of dynamic loading to which a mechanical system is exposed. Relaxation experiments on materials were performed at different isobaric and isothermal states to construct mastercurves, the time-temperature-pressure interrelation was modeled using the Fillers-Moonan-Tschoegl model. Dynamic material functions, related to isolation stiffness and energy absorption, were determined with the Schwarzl approximation. An increase of stiffness and energy absorption at selected hydrostatic pressure, compared to its stiffness and energy absorption at ambient conditions, is represented with $\kappa_{k}(p, \omega)$, defining the increase of stiffness and $\kappa_{d}(p, \omega)$, defining the increase of energy absorption. The study showed that close to the glassy state, moduli of 1190A and 1195D is about 6-9 times higher compared to 1175A, whereas, their properties at ambient conditions are for all practical purposes the same. TPU 1190A turns out to be most sensitive to pressure: at $300 \mathrm{MPa}$ its properties are shifted for 5.5 decades, while for $1195 \mathrm{D}$ and $1175 \mathrm{~A}$ this shift is only 3.5 and 1.5 decades, respectively. In conclusion, the stiffness and energy absorption of isolation may be increased with pressure for about 100 times for 1190A and 1195D, and for about 10 times for $1175 \mathrm{~A}$.

\footnotetext{
${ }^{a)}$ Dedicated to dr. David Binding for his contributions to the field of Rheology.

b) University of Ljubljana, Faculty of Mechanical Engineering, Slovenia, corr. author: marko.bek@fs.uni-lj.si

c) Karlsruhe Institute of Technology, Faculty of Mechanical Engineering, Germany
} 


\section{INTRODUCTION}

Negative effects of vibration and noise on people as well as on performance and reliability of machines and devices are well-known facts ${ }^{1,2}$. Obviously, the best solution is to avoid vibration problem in the first place, however this is often not possible and vibration control is necessary to provide vibration protection. The role of vibration protection is to minimize the vibration transmission between the source and surrounding (receiver). The effectiveness of vibration isolation is determined by vibration parameters: mass, stiffness and damping of an isolator ${ }^{3}$.

Metals and polymers are materials most often used for this purpose. Polymers are used due to their good damping properties through viscous mechanisms, low specific density and good design and production flexibility ${ }^{4}$. On the other hand, also metals exhibit some damping resulting from dislocations, phase boundaries, grain boundaries ${ }^{3}$, and damping arising in structures with complex geometry, resulting from the interplay between the macroscopic vibrations and wave propagation. Comparing metallic and polymeric materials used for damping, shows that polymers exhibit far better damping, however they suffer from low stiffness $^{5}$. This is one of the reasons why metals are still often used for vibration protection, for example in foundation of heavy machinery that operates at constant excitation frequency.

However, for systems that function at or near resonance we need damping elements that exhibits as high energy dissipation (absorption) as possible. In such applications using polymeric materials is the only option.

The selection of a particular polymeric material for vibration control will depend on vibrating system, operating conditions and environment, particularly temperature, where vibrating system is located, as well as on allowable/desired vibration levels. Comparing the stiffness and damping of different polymeric materials, shows that the two properties are contradictory. Hence, polymers with high damping (energy absorption capability), typically elastomeric materials, have lower stiffness compared to polymers with lower damping, typically thermoplastic polymers ${ }^{6,7}$.

Due to their lower stiffness, polymers with higher damping are usually not used for vibration isolation. In addition, these materials exhibit maximal values of damping (energy absorption) at high frequencies, often at frequencies far away from working range of machines and devices. It becomes clear that, at present, we do not have available solutions allowing 
us to utilize the full damping potential that especially elastomeric materials offer. In order to overcome these drawbacks of polymeric materials (limited stiffness and maximal damping at high frequencies), two main challenges need to be addressed: (i) stiffness of a material should be increased and (ii) its maximal values of damping have to be shifted towards lower frequencies.

Combining high stiffness with high damping can be achieved in several ways using different combinations of layered and/or composite structures combining either: (i) metal layers $^{8-11}$ or metal structures ${ }^{12}$ with polymers; (ii) carbon (or other) fibers with polymers ${ }^{13-17}$ and (iii) mixing different materials or adding (nano) particles to the matrix polymeric material $^{7,18-21}$.

Our approach on the other hand, utilizes pure polymeric materials without any modifications to it (no reinforcements or additives) and combines the effect of hydrostatic pressure on polymeric materials and properties of granular materials to obtain desirable stiffness and increased damping.

\section{A. Granular Damping Elements}

The answer to the two challenges are a new generation damping elements which we call Granular damping elements (GDE) which are based on a patented Dissipative bulk and granular systems technology ${ }^{22}$. Patented research-based invention utilizes two scientific findings:

1. Hydrostatic pressure allows for frequency adjustment of the material damping properties.

Using our unique high-pressure apparatus ${ }^{23-25}$ we have shown that exposing polymeric materials to selected hydrostatic pressure causes a shift of mechanical properties to lower frequencies. In other words, high values of damping (that are usually located at higher frequencies) are shifted towards lower frequencies without hampering materials damping (energy absorption) properties. Hence, by selecting a proper (elastomeric) material with high damping properties and exposing it to a selected hydrostatic pressure, we can match the frequency range where material exhibits its maximal dissipation

properties with the excitation (resonance) frequency range of a vibrating structure. 
Using this principle, the material energy dissipation properties can be enhanced for several orders of magnitude.

Unfortunately, pressures required for substantial change of damping properties may be quite high i.e., from $50-100 M P a$ or even higher ${ }^{24,26-28}$. Exposing bulk materials to such pressure levels in uniaxial compression will inevitably lead to appearance of cracks due to excessive accompanying shear stresses, and eventually to material failure.

We have solved this problem with an inventive solution described below.

\section{Improving granular material flow properties for their self-pressurization.}

We have realized that granular materials with proper particle size-distribution may exhibit a flow-like behavior while maintaining all properties of a bulk material. To study the flowability of granular materials we have developed GFA apparatus (Granular Friction Analyzer $)^{29}$. The apparatus allows studying the ability of granular materials to flow in case when driving force is high (hydrostatic) pressure. The GFA is composed of a hollow cylinder (with one of its ends closed) that is loaded with granular material. Once the cylinder is filled with particles, a force is applied by means of a piston; such force will induce a pressure inside the cylinder that induces elastic deformations in the axial and tangential directions of the external wall of the cylinder. The strain in the external face of the cylinder can be measured experimentally, e.g., by means of a strain gages setup, or optically. From the measured strains, we can determine the pressure profile along the length of the cylinder and relate such profile with the flowability of granular materials. If the cylinder would be filled with a Newtonian fluid the internal pressure throughout the cylinder would be uniform and the strain in the external face of the cylinder would be the same along the length of the cylinder. In this context, one expects that depending on the flow properties of granular materials, the pressure will be transmitted in a different way along the cylinder, thus allowing us to obtain a method for flowability characterization ${ }^{29}$.

Based on the studies of granular materials flowability we have concluded that polymeric granular materials with proper particles size-distribution may be used as a pressurizing media (similar as air in tires) to impose hydrostatic pressure on themselves (i.e., self-pressurization) and, as a result, change frequency dependence of their own damping properties. With 


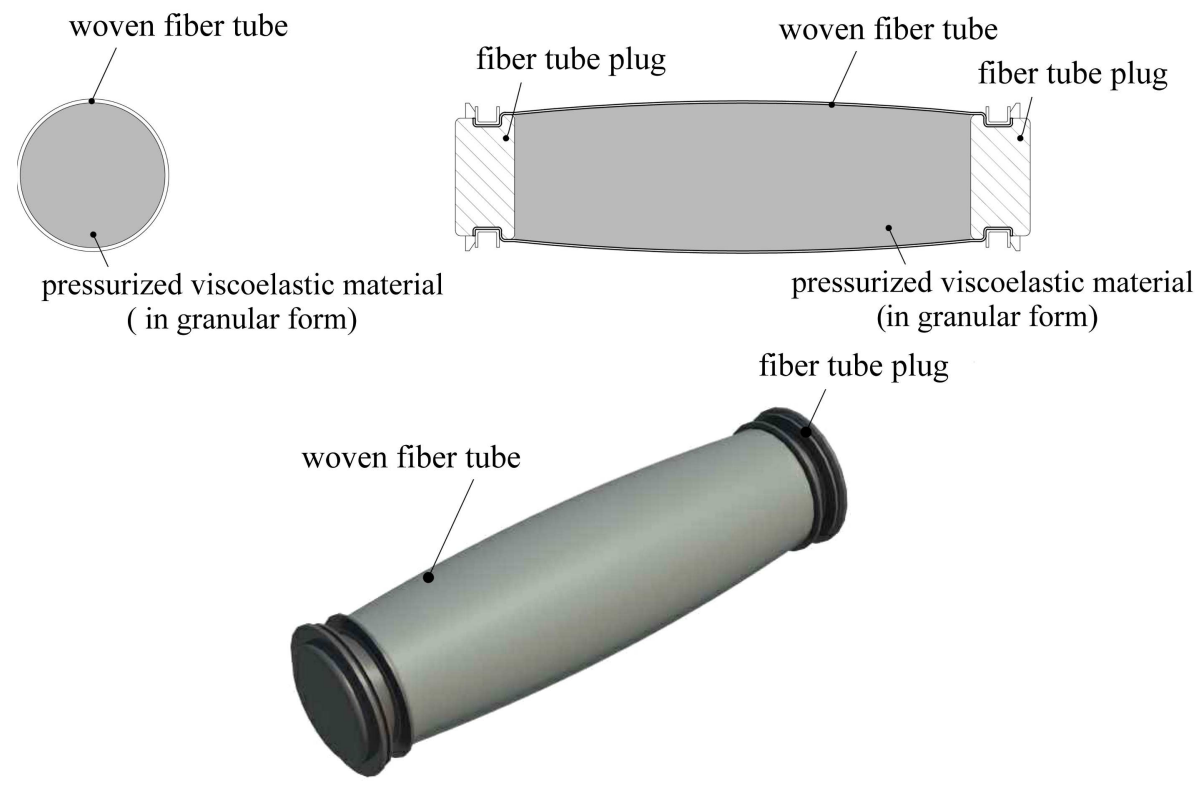

FIG. 1. Schematic presentation of Granular damping element (GDE).

a proper adjustment of the hydrostatic pressure, we can also adjust the stiffness of the damping element (again, similar as with air in tires). Since energy dissipation of a damping element is proportional to the volume of used granular materials, whereas the stiffness in addition depends on geometry of a damping element, the two parameters (damping and stiffness) may be adjusted independently.

Considering this, polymeric Granular damping elements (GDE), were developed. GDE consists of a container, made of woven basalt, carbon or glass fibers, which is filled and pressurized with polymeric granular materials with multi-modal particles size-distribution. Due to the flow-like behavior of granulated polymers, the generated pressure within the container will be hydrostatic and will act on polymeric particles themselves, and consequently modify frequency dependence of their energy absorption properties. Hence, with a proper pressurization we may shift the material energy absorption maximum to any desired frequency. A sketch of such Granular damping element (GDE) is shown Fig.1, whereas Fig.2 schematically presents its working principle. Described GDE elements differ from the similar elements, devices and patents ${ }^{30-33}$ that use sand ${ }^{34,35}$, granulated polymers ${ }^{36-39}$ or metals ${ }^{40-45}$ for enhanced damping, since in our case enhanced damping is not only the result of friction between particles but also result of changed material properties.

Let us assume that a vibrating structure is equipped with a damping element made 


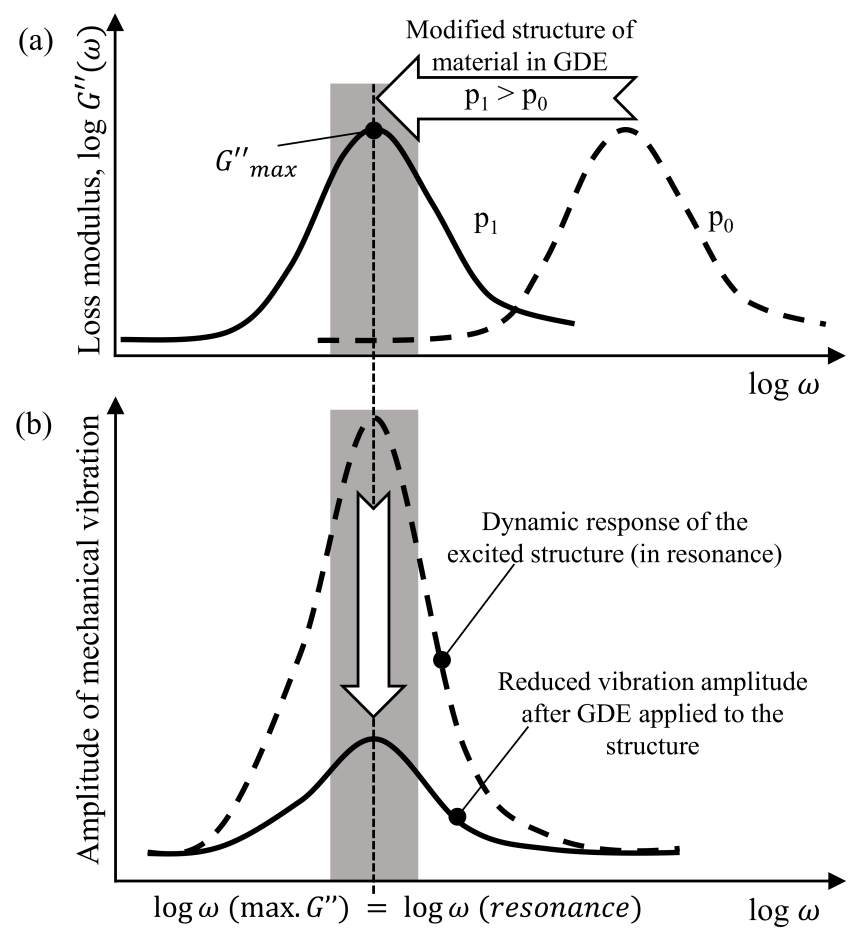

FIG. 2. Working principle behind GDE elements.

of a polymeric material of which its frequency dependent damping properties at ambient pressure $p_{0}$, expressed with the loss modulus $G^{\prime \prime}(\omega)$, are shown in Fig.2(a) as a dashed line. In the frequency range where the structure is in resonance, indicated as a shadowed area, the damping properties of this material are low. Consequently, the structure vibration amplitudes in the resonance frequency range will be large, as shown with a dashed line in Fig.2(b). Now, if we take the very same material in a granular form with a proper particlessize distribution and self-pressurize it within a woven container, we obtain the new GDE damping element with the ultimate damping properties. This is achieved by selecting a proper hydrostatic pressure $p_{1}$ within the container that shifts the loss modulus maximum to the resonance frequency range of the structure, as shown in Fig.2(a) with a solid line. As a result, the vibration amplitudes of the structure will substantially diminish, as shown as a solid line in Fig.2(b).

To summarize, for vibration and/or impact control, where high damping is necessary, high values of the loss modulus $G^{\prime \prime}(\omega)$ are needed since the loss modulus is related to dissipated energy. To fully utilize the damping properties of a polymeric material, the frequency range of its loss modulus maximum $G^{\prime \prime}{ }_{\max }(\omega)$ should be matched with an excitation frequency or an impact rate imposed to a mechanical system. As mentioned before, this may be achieved 
by exposing the elastomeric granular material to a properly selected hydrostatic pressure and adjust the frequency at which the material exhibits maximum energy dissipation i.e., $G^{\prime \prime}{ }_{\max }(\omega)$ such that it matches the resonance frequency of the vibrating structure. The described approach allows ultimate utilization of damping properties of all existing and/or newly developed polymeric materials of which its maximum damping frequency is located at higher frequencies than the resonance frequency range of an observed dynamic system.

To this end, the goal of this paper is to examine the pressure and frequency dependence of three polyether-based thermoplastic polyurethane (TPU) materials on vibration isolation. The investigated materials are from the Elastollan ${ }^{\circledR} 11$ series $^{46}$ i.e., (i) $1190 \mathrm{~A}$, (ii) $1175 \mathrm{~A}$, and (iii) 1195D, manufactured by BASF. Within this paper, properties of TPU materials in solid state are investigated, which after granulation are potential candidates for producing the new generation GDE damping elements.

\section{BACKGROUND}

The background section reviews the underlying knowledge relevant for the experimental investigation of the three materials to be used in a new generation vibration isolation systems. It is sub-divided into several sections, starting with a brief review of the effect of pressure, followed by the description of high-pressure CMS apparatus ${ }^{47}$, the new standardized procedure for generating unique master curves and selected methodology for interconverting material functions between the time and frequency domain. The latter is needed because the shear relaxation experiments under pressure have been performed in time-domain and had to be converted into the frequency domain to obtain information on the storage modulus $G^{\prime}(\omega)$, responsible for vibration isolation stiffness, and information on the loss modulus $G^{\prime \prime}(\omega)$ which represents the material's capability to dissipate mechanical energy.

\section{A. The effect of pressure}

A comprehensive review of the effect of pressure and temperature on time-dependent behavior of polymeric materials is given in Tschoegl, et. $\mathrm{al}^{48}$, here we will briefly review only those facts that are relevant to the present paper.

The effect of pressure on macroscopic time-dependent behavior of polymeric materials is, 
in principle, analogous but opposite to that of temperature. As it is well known, exposing viscoelastic materials to lower temperatures (compared to a reference temperature) hinders the mobility of molecular chains in the amorphous phase, which is exhibited through the extension of the material creep and relaxation time scales ${ }^{25,49}$. On the other hand, exposing viscoelastic material to higher temperatures (compared to a reference temperature) increases the mobility of the molecular chains, which is now exhibited as reduction of the material relaxation (and creep) time scales. Pressure has similar, though opposite effect on material macroscopic time-dependent mechanical properties as temperature. When viscoelastic materials are exposed to high (hydrostatic) pressures the mobility of polymer chains is hindered (similarly as exposing material to low temperatures). On the macro scale, this is exhibited through the extension of the material relaxation (and creep) time scales. Hence, under hydrostatic pressure the viscoelastic relaxation and retardation times of solid polymers increase. The opposite happens when material is exposed to decreased pressure (relative to a higher reference pressure), viscoelastic relaxation and retardation times of polymers decrease ${ }^{25,48}$. Of course, it is obvious that the ambient pressure is essentially the lowest pressure to which material may be exposed.

Since frequency and time domain are interrelated, pressure also affects dynamic material properties i.e., storage modulus $G^{\prime}(\omega)$, loss modulus $G^{\prime \prime}(\omega)$ and damping ratio $\delta(\omega)$. Due to the same molecular mechanisms, increased pressure causes the dynamic properties to shift towards lower frequencies (compared to reference values) and vice-versa; decreased pressure, relative to a higher reference pressure, causes properties to shift towards higher frequencies.

There are several different models for mathematical description of the effect of pressure and temperature ${ }^{48}$, in our case we have used the so called FMT model, proposed by Fillers, Moonan and Tschoegl ${ }^{26-28}$. The FMT model can be viewed as an extension of the WLF equation to account for the effect of pressure in addition to that of temperature. The shift factor as function of temperature and pressure is given in the form:

$$
\log a_{T, p}=-\frac{c_{1}^{00}\left[T-T_{0}-\theta(p)\right]}{c_{2}^{00}(p)+T-T_{0}-\theta(p)}
$$

where

$$
\theta(p)=c_{3}^{0}(p) \ln \left(\frac{1+c_{4}^{0} p}{1+c_{4}^{0} p_{0}}\right)-c_{5}^{0}(p) \ln \left(\frac{1+c_{6}^{0} p}{1+c_{6}^{0} p_{0}}\right)
$$


and c's follow as

$$
\begin{gathered}
c_{1}^{00}=B / 2.303 f_{0}, \\
c_{2}^{00}=f_{0} / \alpha_{f}(p), \\
c_{3}^{0}(p)=1 / k_{e} \alpha_{f}(p), \\
c_{4}^{0}=k_{e} / K_{e}^{*}, \\
c_{5}^{0}(p)=1 / k_{\phi} \alpha_{f}(p), \\
c_{6}^{0}=k_{\phi} / K_{\phi}^{*},
\end{gathered}
$$

The 00 superscript indicates that the parameter is referred to the reference temperature $T_{0}$ (first place) and to the reference pressure $p_{0}$ (second place). A single 0 superscript refers to the reference temperature only. The ${ }^{*}$ superscript refers to zero (in practice, atmospheric) pressure, while subscripts $e$ and $f$ stand for entire- and free- volume, respectively.

Eq. 1 is the Fillers-Moonan-Tschoegl (FMT) equation. $K_{e}^{*}$ and $k_{e}$, and thus $c_{4}^{0}$, can be determined from separate volume-pressure measurements through a fit to the the Murnughan equation of state $^{50}$

$$
\ln \frac{V}{V_{0}}=-\frac{1}{k_{e}} \ln \left[\frac{K_{e}^{*}(T)+k_{e} p}{K_{e}^{*}(T)+k_{e} p_{0}}\right]
$$

by a non-linear least-squares procedure. In Eqs.1-9, $\alpha_{f}$ denotes expansivity (i.e., the isobaric cubic thermal expansion coefficient) of the fractional free volume, $f_{0}=f\left(p_{0}\right)$ is fractional free volume at reference pressure, $K_{e}^{*}(T)=\left.K_{e}(T)\right|_{p=0}$ is the bulk modulus at atmospheric pressure and $k_{e}$ is a proportionality constant deemed independent of either pressure or temperature.

Described facts on the effect of pressure demonstrate that hydrostatic pressure may be used for adjustments of the energy absorption maximum to a desired frequency. However, since vibration isolation usually operates at ambient conditions, pressure may be only increased. The pressure induced shifting is therefore possible from higher toward the lower frequencies only. Hence, for the GDE damping elements we may use only those polymeric materials which energy absorption maxima are located at frequencies that are much higher than the frequency at which vibrations take place. The three materials analyzed here fulfill this condition. 


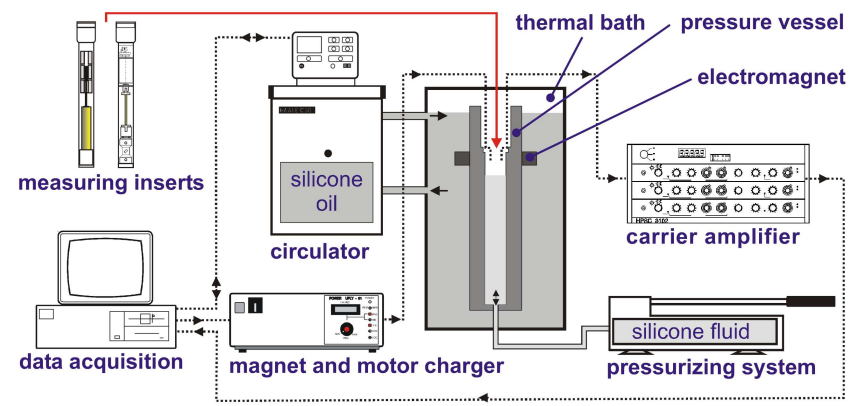

FIG. 3. Schematic representation of CMS apparatus. Reproduced from Experimental mechanics, A measuring system for bulk and shear characterization of polymers, 46, 2006, 429, I. Emri and T. Prodan, Society for Experimental Mechanics 2006. With permission of Springer.

\section{B. High-pressure CMS apparatus}

All experiments were performed on a CMS apparatus ${ }^{23-25}$. CMS allows measurements of shear relaxation and bulk creep compliance properties of polymers in solid state, simultaneously subjected to temperatures ranging from $-40^{\circ} \mathrm{C}$ to $+120^{\circ} \mathrm{C}$, and hydrostatic pressures ranging from atmospheric to $500 M P a$. The apparatus is shown schematically in Fig.3. The pressure is generated by the pressurizing system using silicone oil. The pressure vessel is contained within the thermal bath, where another silicone oil circulates from the circulator, used for precise temperature control. The apparatus utilizes two separate measuring inserts, which can be inserted into the pressure vessel, the relaxometer and the dilatometer, both shown in Fig.4. Signals from the measuring inserts pass through the carrier amplifier prior to being collected in digital format by the data acquisition system.

The magnet and motor charger supplies current to the electromagnet, which activates the measurement. The same charger also supplies current to the electric motor of the relaxometer, shown in Fig.4(a), which pre-loads the spring that then applies the desired torsional deformation (angular displacement), to the specimen. Specimens can be simultaneously subjected to pressures of up to $500 M P a$ with a precision of $\pm 0.1 M P a$, and to temperatures ranging from $-40^{\circ} \mathrm{C}$ to $+120^{\circ} \mathrm{C}$ with a precision of $\pm 0.01^{\circ} \mathrm{C}$.

The relaxometer insert, shown in Fig.4(a), measures the shear relaxation modulus by applying a constant torsional strain to a cylindrical specimen, and by monitoring the induced moment as a function of time. The specimen diameter can range from $2 \mathrm{~mm}$ to $12 \mathrm{~mm}$, while its length may vary from $38 \mathrm{~mm}$ to $60 \mathrm{~mm}$. The two main parts of the insert are the loading device, and the load cell. The loading device applies a torsional strain by twisting the 


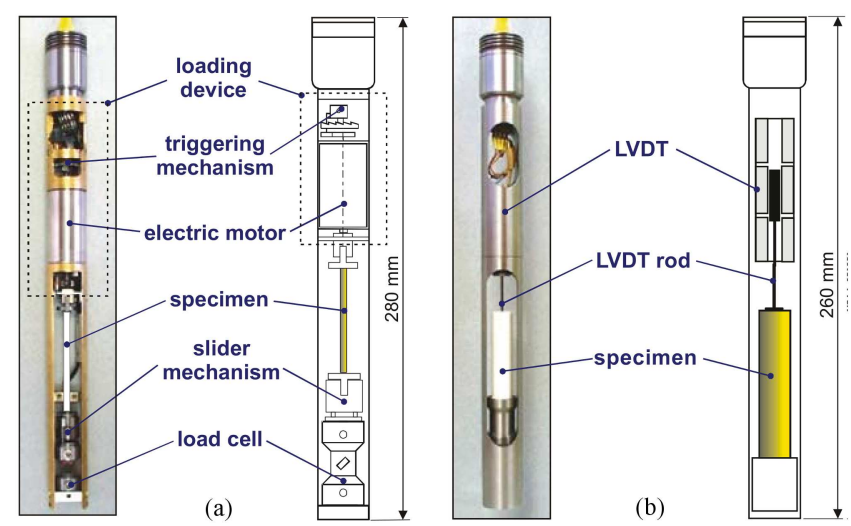

FIG. 4. Relaxometer (a) and dilatometer (b) inserts with main components. Reproduced from Experimental mechanics, A measuring system for bulk and shear characterization of polymers, 46, 2006, 429, I. Emri and T. Prodan, Society for Experimental Mechanics 2006. With permission of Springer.

specimen for a few degrees in approximately 0.01 seconds (depending on the initial stiffness of the specimen). To induce the deformation of the specimen, the electric motor is used to first pre-load an Archimedes spring. Once twisted, the spring is kept in its pre-loaded position by a rack-and-pawl mechanism, which is activated with the electromagnet, mounted outside the pressure vessel (see Fig.3). The latter pulls the pawl out of the rack, and the energy of the spring deforms the specimen to a pre-determined angle, which is typically around 2 degrees. The induced moment is then measured by the load cell, which is attached to the slider mechanism to compensate for possible changes in the length of the specimen resulting from various temperature and pressure conditions, as well as from the pointing effect (shortening of the specimen caused by a torsional deformation). After the shear relaxation measurement is complete, the electric motor brings the specimen to its original un-deformed state, while maintaining the pressure vessel fully pressurized. The relaxometer can measure shear relaxation moduli in the range of $10^{-2}-10^{4} \mathrm{MPa}$.

The shear relaxation modulus $G(t)$ is the ratio of the time-dependent shear stress $\tau(t)$ over a fixed and constant shear strain $\gamma_{0}$ :

$$
G(t)=\frac{\tau(t)}{\gamma_{0}}=\frac{32 L_{0} M(t)}{\pi D_{0}^{4} \theta_{0}} .
$$

Here $M(t)$ is a decaying moment measured over time with a load cell, $\theta_{0}$ is an initial angular deformation of the specimen induced by the spring, as described above, and $D_{0}$ and $L_{0}$ are the specimen diameter and effective length (distance between the two grips) respectively. 
The dilatometer insert, shown in Fig.4(b), is used to measure bulk properties such as the: bulk creep compliance, $B(t, T, p)$; equilibrium bulk creep compliance, $B(T, p)=$ $B(t \rightarrow \infty, T, p)$; equilibrium bulk modulus, $K(T, p)=K(t \rightarrow \infty, T, p)=1 / B(T, p)$; specific volume $v(T, p)=v(t \rightarrow \infty, T, p)$, and thermal (equilibrium) expansion coefficient, $\beta(T, p)=\beta(t \rightarrow \infty, T, p)$. The measurements are performed by monitoring the volume change of the specimen, which results from the imposed changes in pressure and/or temperature, by measuring the change in length of the specimen, $L(t, T, p)$, with the aid of a built in Linear Variable Differential Transformer (LVDT). The volume estimate can be considered accurate if the change in volume is small (up to a few percent) and the material is isotropic. Specimens for the dilatometer can be up to $16 \mathrm{~mm}$ in diameter and from 40 to $60 \mathrm{~mm}$ in length. The absolute volume measurement error is about $0.1 \%$, while the relative error is $0.05 \%$.

Within this research the specific volume $v(T, p)$, measurements were needed for determination of FMT constants (non-linear least-squares fitting of Eq.9). The specific volume is defined as a ratio between the specimen volume $V$ and its mass $m$ :

$$
v(T, p)=\frac{V\left(t_{\infty}, T, p\right)}{m}=\frac{\pi D_{0}^{2}}{4 m L_{0}^{2}}\left(L_{0}+\Delta L\right)^{3},
$$

where $L_{0}$ and $D_{0}$ are the initial length and diameter of a specimen and $\Delta L$ is the change of the length of the specimen induced by temperature/pressure changes. Since the change of volume is small we assumed that the relative change in length $L$, equals the relative change in diameter

$$
\frac{D}{D_{0}} \approx \frac{L}{L_{0}} \approx \frac{L_{0}+\Delta L}{L_{0}} \rightarrow D=\frac{\left(L_{0}+\Delta L\right) D_{0}}{L_{0}}
$$

Similarly, we find for the volume of a cylindrical specimen:

$$
V=\frac{\pi D_{0}^{2}}{4 L_{0}^{2}}\left(L_{0}+\Delta L\right)^{3}
$$

\section{Construction of mastercurves using CFS methodology}

As will be explained in the experimental section, the experiments were performed at several different temperatures and pressures within an experimental window of $10^{3}$ seconds to obtain the response function segments, which had to be shifted according to the time- 


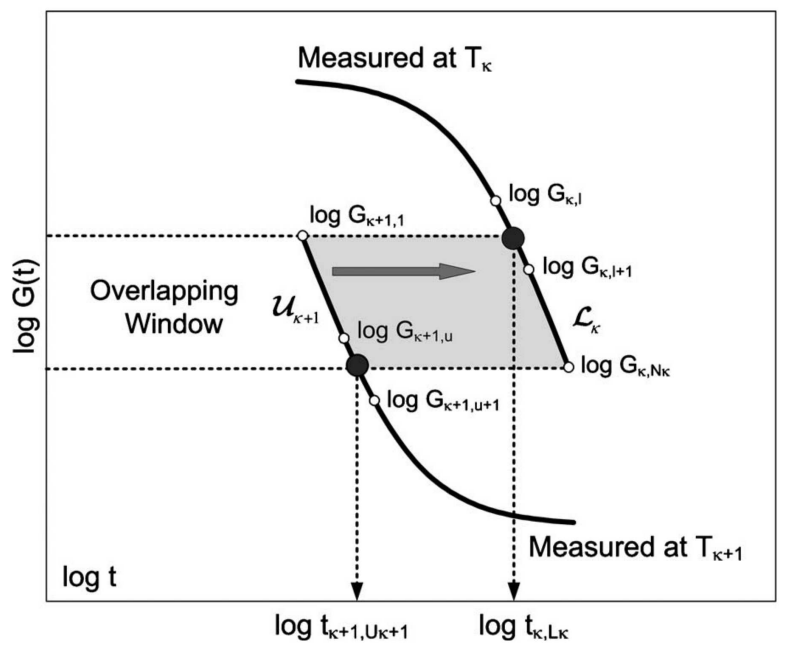

FIG. 5. Schematics of the shifting procedure. Reproduced from M. Gergesova, B. Zupancic, I. Saprunov, and I. Emri, Journal of Rheology, Vol. 55, 1, (2011); used in accordance with the Creative Commons Attribution (CC BY) license (https://creativecommons.org/licenses/by/4.0/).

temperature-pressure superposition principle ${ }^{48}$ to obtain the corresponding mastercurve. Instead of commonly used hand shifting, the shifting was done by using the close-formshifting (CFS) $)^{51,52}$ algorithm, which leads to a unique objective mastercurve. The CFS shifting procedure, which is becoming the new ISO standard (ISO/DIS 18437-6:2017), is briefly summarized below.

Let the temperature $T_{k}$ be the reference temperature, and the corresponding data measured at these conditions the reference segment of the master curve,

$$
G\left(T_{k}, t\right)=\left\{\log G_{k, i}, \log t_{k, i} ; i=1,2, \ldots, N_{k}\right\}
$$

To construct the master curve at the selected temperature $T_{k}$ we need to shift all segments measured at $T_{j}>T_{k}$ to the right, and the remaining segments, measured at $T_{j}<T_{k}$ to the left along the logarithmic time-axis, so as to obtain a smooth mastercurve, as schematically shown in Fig.5. The shifting must be executed sequentially, starting with segments measured at $T_{k+1}>T_{k}$, and $T_{k-1}<T_{k}$, until all the segments are brought together.

The set of temperatures, $\left\{T_{j}, j=1,2, \ldots, M\right\}$, at which relaxation experiments were performed should be selected such that segments measured at two consecutive temperatures, e.g., $T_{k}$, and $T_{k+1}$ will overlap sufficiently to allow effective construction of the master curve. The common rule of thumb is that this overlapping should be at least one logarithmic decade 
of time.

The first datum point of the segment $(k+1)$ i.e., $\log G_{k+1,1}$, and the last datum point of the segment $(k)$ i.e., $\log G_{k, N_{k}}$ represent strengths of the first and the last datum points in the window where the two segments overlap, as shown in Fig.5.

The overlapping sets of two segments we denote as

$$
U_{k+1}=\left\{\log G_{k+1, n}, \log t_{k+1, n} ; n=1,2, \ldots, U_{k+1}\right\},
$$

and

$$
L_{k}=\left\{\log G_{k, n}, \log t_{k, n} ; n=L_{k}, L_{k}+1, \ldots, N_{k}\right\} .
$$

The location of the last datum point of the set $U_{k+1}$ i.e., $\log G_{k+1, U_{k+1}}=\log G_{k, N_{k}}$, may be expressed as

$$
\begin{aligned}
\log t_{k+1, U_{k+1}}= & \log t_{k+1, u}+\left(\log G_{k+1, u}-\log G_{k+1, U_{k+1}}\right) \\
& \times \frac{\log t_{k+1, u+1}-\log t_{k+1, u}}{\log G_{k+1, u}-\log G_{k+1, u+1}}
\end{aligned}
$$

Whereas the location of the first datum point of the set $L_{k}$ i.e., $\log G_{k, L_{k}}=\log G_{k+1,1}$, is given in the form,

$$
\begin{aligned}
\log t_{k, L_{k}}= & \log t_{k, l}+\left(\log G_{k, l}-\log G_{k, L_{k}}\right) \\
& \times \frac{\log t_{k, l+1}-\log t_{k, l}}{\log G_{k, l}-\log G_{k, l+1}} .
\end{aligned}
$$

To construct the master curve at the selected temperature $T_{k}$ we need to shift the segment measured at $T_{k+1}$ to the right along the logarithmic time-axis, to obtain the smooth mastercurve. Thus, in the ideal case, for all data within the Overlapping Window the condition

$$
G_{k}(t)=G_{k+1}\left(t \cdot a_{k+1}\right)
$$

must be fulfilled. Here $a_{k+1}$ denotes time-temperature-pressure shift factor corresponding to the temperature $T_{k+1}$. We may intuitively see that an optimal overlapping of the two curves is obtained when the shaded area between the two curves is equal to zero, see Fig.5. 
Hence,

$$
A=A_{k}-A_{k+1}=0
$$

where

$$
\begin{aligned}
A_{k+1}=\sum_{n=1}^{U_{k+1}-1} & {\left[\left(\frac{\left(\log t_{k+1, n+1}+\log a_{k+1}\right)}{2}\right)\right.} \\
& \left.\times \frac{+\left(\log t_{k+1, n}+\log a_{k+1}\right)}{2}\right) \\
& \left.\times\left(\log G_{k+1, n+1}-\log G_{k+1, n}\right)\right],
\end{aligned}
$$

and

$$
A_{k}=\sum_{n=L_{k}}^{N_{k}-1}\left[\frac{\log t_{k, n+1}+\log t_{k, n}}{2}\left(\log G_{k, n+1}-\log G_{k, n}\right)\right]
$$

Combining equations (17) - (22), we may calculate the shift factor belonging to the segment measured at $t_{k+1}$

$$
\begin{aligned}
\log a_{k+1}= & \frac{A_{k}-\sum_{n=1}^{U_{k+1}-1}\left[\frac{\log t_{k+1, n+1}+\log t_{k+1, n}}{2}\right.}{\log G_{k, N_{k}}-\log G_{k+1,1}} \\
& \times \frac{\left.\left(\log G_{k+1, n+1}-\log G_{k+1, n}\right)\right]}{\log G_{k, N_{k}}-\log G_{k+1,1}} .
\end{aligned}
$$

This algorithm is valid for shifting the segments measured at $T_{j}>T_{k}$. For shifting those segments that were measured at $T_{j}<T_{k}$ we need to modify the algorithm as follows,

$$
\begin{aligned}
\log a_{k-1}= & \frac{A_{k}-\sum_{n=L_{k}-1}^{N_{k-1}-1}\left[\frac{\log t_{k-1, n+1}+\log t_{k-1, n}}{2}\right.}{\log G_{k-1, N_{k-1}}-\log G_{k, 1}} \\
& \times \frac{\left.\left(\log G_{k-1, n+1}-\log G_{k-1, n}\right)\right]}{\log G_{k-1, N_{k-1}}-\log G_{k, 1}},
\end{aligned}
$$

where

$$
A_{k}=\sum_{U_{k}-1}^{n=1}\left[\frac{\log t_{k, n+1}+\log t_{k, n}}{2} \cdot\left(\log G_{k, n+1}-\log G_{k, n}\right)\right]
$$


We have used the above CFS approach to generate the unique, person-independent master curves presented in this paper. The CFS software may be freely downloaded at: http: //standards.iso.org/iso/18437/-6/ed-1/en.

\section{Interconversion between time- and frequency-domain material functions}

Pressure dependence characterization was performed in time-domain as relaxation experiments. Details are presented in experimental section (Section III). The frequency dependent material functions i.e., the storage modulus $G^{\prime}(\omega)$ and the loss modulus $G^{\prime \prime}(\omega)$, where then

obtained via the interconversion. For the case of shear relaxation $G(t)$, and storage $G^{\prime}(\omega)$ and loss $G^{\prime \prime}(\omega)$ modulus, the interrelation is given through the inverse Fourier transom ${ }^{53}$ as:

$$
\begin{aligned}
G(t) & =\mathcal{F}^{-1}\left[G^{*}(\omega) / i \omega ; t\right] \\
& =\frac{1}{2 \pi} \int_{-\infty}^{\infty} \frac{G^{*}(\omega)}{i \omega} \exp (i \omega t) d \omega
\end{aligned}
$$

where $i=\sqrt{-1}$, and $G^{*}(\omega)$ is the complex modulus, composed of storage and loss modulus:

$$
G^{*}(\omega)=G^{\prime}(\omega)+i G^{\prime \prime}(\omega)
$$

However, there are several other possible ways to interconvert material functions from the time to frequency domain and vice versa ${ }^{54}$. In our case we have used an approximate method proposed by Schwarzl ${ }^{55}$. In this case the interrelation between the storage $G^{\prime}(\omega)$ and the loss $G^{\prime \prime}(\omega)$ modulus and the shear relaxation $G(t)$ is given as:

$$
\begin{aligned}
G^{\prime}(\omega) & \cong 0.143 G(8 t)-0.86 G(4 t)+0.674 G(2 t) \\
& +0.842 G(t)+0.001 G(t / 2)+0.101 G(t / 4) \\
& -0.00855 G(t / 8)+\left.0.00855 G(t / 16)\right|_{t=1 / \omega}
\end{aligned}
$$

and

$$
\begin{aligned}
G^{\prime \prime}(\omega) & \cong 0.470 G(4 t)-2.144 G(2 t)+1.476 G(t) \\
& -0.422 G(t / 2)+0.608 G(t / 4) \\
& -0.160 G(t / 8)+\left.0.1722 G(t / 16)\right|_{t=1 / \omega}
\end{aligned}
$$


TABLE I. Extrusion parameters for TPU's on PolyLab HAAKE Rheomex PTW 16 extruder.

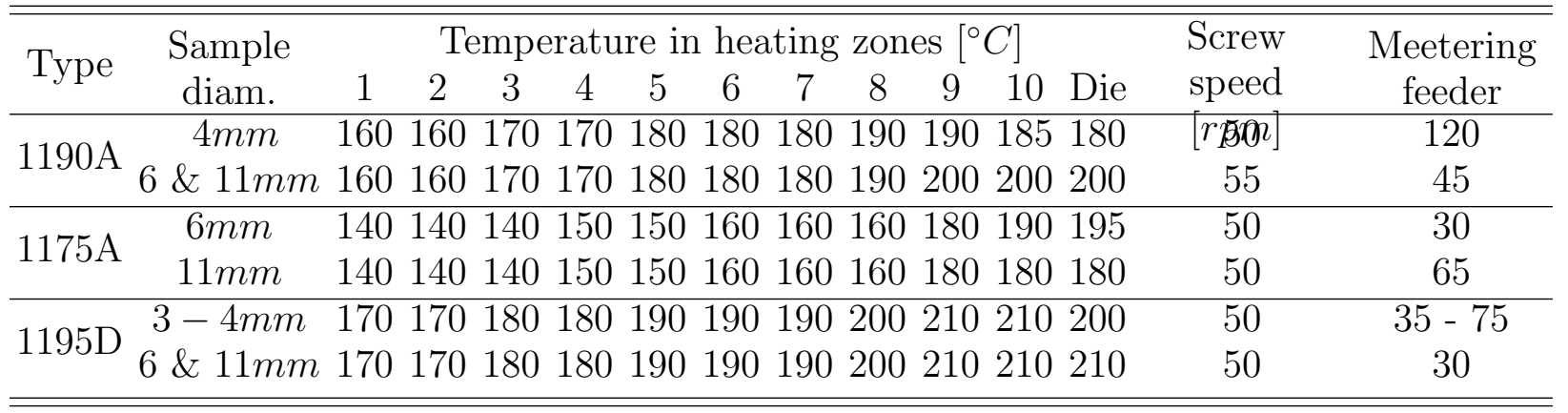

The error analysis which we have performed in the past ${ }^{54}$ showed that this approximate method brings in an error smaller than $2 \%$, which we considered sufficient for the purpose of current investigation.

\section{CHARACTERIZATION OF SELECTED MATERIALS}

We have investigated three polyether-based thermoplastic polyurethane (TPU) materials in solid state from the BASF Elastollan ${ }^{\circledR} 11$ series ${ }^{46}$ i.e., (i) $1190 \mathrm{~A}$, (ii) $1175 \mathrm{~A}$, and (iii) $1195 \mathrm{D}$, that are already used for vibration isolation applications. Since Elastollan ${ }^{\circledR}$ is hygroscopic material, all materials were dried at $100^{\circ} \mathrm{C}$ for 3 hours in a commercial dryer (SP105-C, Kambič, Slovenia), to avoid bubble formation during the extrusion process, which was used for sample preparation.

\section{A. Bulk sample preparation}

Due to measuring limitations of the CMS apparatus (see Section II B), we had to prepare samples with different diameters for measurements at different pressure-temperature boundary conditions. Samples were prepared with a PolyLab HAAKE Rheomex PTW 16 extruder (Thermo Haake, Germany) equipped with two co-rotating $16 \mathrm{~mm}$ screws. Its extruder barrel is divided into ten independent heating zones and a separate die temperature control. Based on experience, we have used the temperature profiles summarized in Tab.I.

Thicker samples $(\sim \phi 6 \mathrm{~mm}$ and $\phi 11 \mathrm{~mm})$ were prepared by extrusion into glass tubes that were coated from the inside with a silicon rubber (Tesacoma, silicone pastry board), to prevent the extruded melt from sticking to the glass. Coated glass tubes were pre-heated 
to $100^{\circ} \mathrm{C}$ before they were filled with materials, and afterwards naturally cooled to ambient conditions. Thinner samples $(\sim \phi 2-4.5 \mathrm{~mm})$ were prepared by continuous extrusion into a water bath. The speed of a subsequent conveyor belt together with the screw speed enabled us to alter diameters of thin samples. After extrusion, the thin samples were let to free hang under their own weight for about 12 hours at ambient conditions, to make them straight.

After extrusion, materials were cut with a razor blade to the specified length and the cut surfaces were finished with a sand paper (using very fine P220 and super fine P1200 sand paper). Diameter of each sample was measured 10 times along its diameter, and its length 5 times, using a digital caliper (Digimatic Caliper Series 500, Mitutoyo, USA). The weight of samples for bulk properties was measured 5 times using a laboratory scale (Mettler Toledo AG245, USA). The averaged values of diameter and length were used to calculate shear relaxation $G(t)$, according to Eq.10, while for bulk measurements also the average weight of samples was taken into account and used for calculating $K_{e}^{*}$ and $k_{e}$, and thus $c_{4}^{0}$ through the Eq.9. Samples were afterwards glued to the metallic sample holders using two component epoxy glue (UHU plus schnellfest 2-K-Epoxidharzkleber, UHU GmbH \& Co KG, Germany), so that they could be placed into the relaxometer insert (Fig.4(a)) or dilatometer insert (Fig.4(b)). The averaged lengths and diameters of samples used for relaxometer measurements are presented in Tab.II, the sample dimensions and mass used for dilatometer measurements are presented in Tab.III.

To remove residual stresses in samples from extrusion, cutting and gluing, all samples were annealed prior to the measurements (this is indicated in Figs.7 and 8 in the following section). The start of this procedure was raising the temperature in the commercial dryer (SP105-C, Kambič SP105-C, Slovenia) from ambient conditions to $90^{\circ} \mathrm{C}$. The temperature was held constant for three hours, followed by slow cooling. Cooling was done in two stages, first stage was done inside the oven to ambient conditions, around $20^{\circ} \mathrm{C}$, and in second stage samples were removed from the oven and placed inside an insulation box (to assure required rate of cooling) that was placed inside a freezer. Using this procedure temperature of $-20^{\circ} \mathrm{C}$ was achieved. During the cooling the average cooling rate was approximately $-0.15^{\circ} \mathrm{C} / \mathrm{min}$ (assuring slow cooling is important in order to minimize the effect of physical aging ${ }^{56}$ ). 
TABLE II. Averaged values of sample dimensions for shear relaxation measurements.

\begin{tabular}{|c|c|c|}
\hline Type & dimensions, $d \times l[\mathrm{~mm}]$ & boundary conditions \\
\hline \multirow{6}{*}{ 1190A } & $6.400 \times 57.703$ & \multirow{3}{*}{$\begin{array}{c}T=-20-30^{\circ} \mathrm{C} \\
p=0.1 M P a\end{array}$} \\
\hline & $5.921 \times 57.854$ & \\
\hline & $5.528 \times 59.012$ & \\
\hline & $4.160 \times 56.966$ & \multirow{3}{*}{$\begin{array}{c}T=-20^{\circ} \mathrm{C} \\
p=0.1-300 M P a\end{array}$} \\
\hline & $3.915 \times 42.586$ & \\
\hline & $3.748 \times 39.714$ & \\
\hline \multirow{6}{*}{$1175 \mathrm{~A}$} & $6.050 \times 53.934$ & \multirow{3}{*}{$\begin{array}{c}T=-20^{\circ} \mathrm{C} \\
p=0.1-300 M P a\end{array}$} \\
\hline & $6.111 \times 54.031$ & \\
\hline & $6.117 \times 53.854$ & \\
\hline & $11.854 \times 58.708$ & \multirow{3}{*}{$\begin{array}{c}T=-20-60^{\circ} \mathrm{C} \\
p=0.1 M P a\end{array}$} \\
\hline & $11.479 \times 58.613$ & \\
\hline & $11.563 \times 58.21$ & \\
\hline \multirow{8}{*}{$1195 \mathrm{D}$} & $3.054 \times 57.468$ & \multirow{3}{*}{$\begin{array}{c}T=-20-10^{\circ} \mathrm{C} \\
p=0.1 M P a\end{array}$} \\
\hline & $3.018 \times 59.962$ & \\
\hline & $4.778 \times 59.783$ & \\
\hline & $6.592 \times 60.005$ & \multirow{3}{*}{$\begin{array}{c}T=20-60^{\circ} \mathrm{C} \\
p=0.1 M P a\end{array}$} \\
\hline & $6.864 \times 58.950$ & \\
\hline & $6.773 \times 58.920$ & \\
\hline & $4.629 \times 59.258$ & $T=-20^{\circ} \mathrm{C}$ \\
\hline & $4.592 \times 57.748$ & $p=0.1-300 M P a$ \\
\hline
\end{tabular}

TABLE III. Averaged values of sample dimensions and mass for bulk measurements.

\begin{tabular}{ccr}
\hline \hline Type & dimensions, $d \times l[\mathrm{~mm}]$ & mass $[\mathrm{g}]$ \\
\hline \multirow{2}{*}{$1190 \mathrm{~A}$} & $11.831 \times 42.952$ & 5.2848 \\
& $11.590 \times 46.451$ & 5.4939 \\
& $11.743 \times 47.533$ & 5.7628 \\
\hline \multirow{2}{*}{$1175 \mathrm{~A}$} & $11.717 \times 49.552$ & 6.4141 \\
& $11.788 \times 48.643$ & 6.2434 \\
& $11.876 \times 49.630$ & 6.1127 \\
\hline \multirow{2}{*}{$1195 \mathrm{D}$} & $11.708 \times 49.085$ & 6.1012 \\
& $11.645 \times 49.620$ & 6.1127 \\
\hline \hline
\end{tabular}

\section{B. Loading profiles for shear relaxation and dilatometry experiments}

After the sample preparation phase, samples were tested using the previously described CMS apparatus, using relaxometer and dilatometer insert (Figs.4(a) and 4(b))

For obtaining the shear relaxation properties, experiments were performed at two dif- 


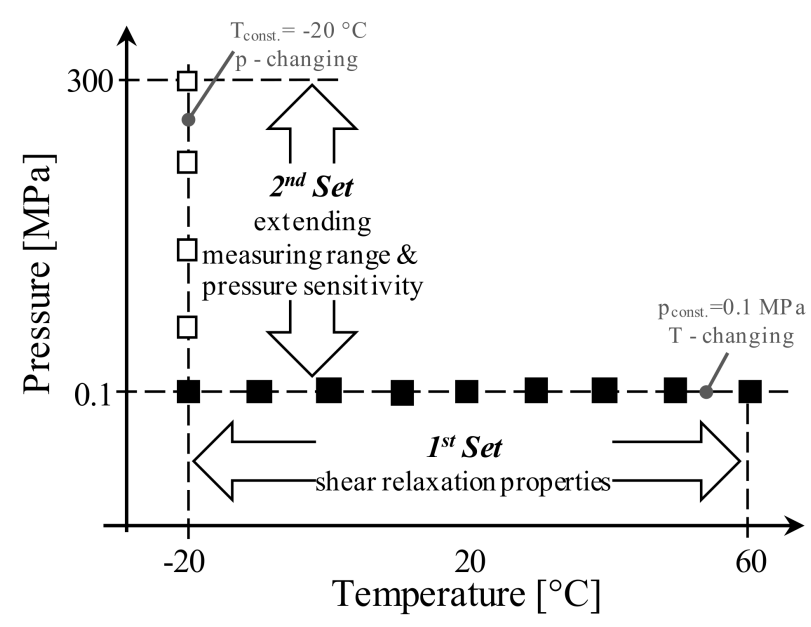

FIG. 6. Boundary conditions for determining shear relaxation properties and pressure sensitivity of selected materials.

ferent sets of boundary conditions. The $1^{\text {st }}$ Set of experiments was performed at isobaric conditions (at constant pressure) at $p=0.1 M P a$, at different constant temperatures between -20 to $60^{\circ} \mathrm{C}$. The exact temperatures are provided within diagrams (Fig.7). For obtaining the pressure sensitivity of measured materials and to expand the measuring range of shear relaxation $G(t)$, the $2^{\text {nd }}$ Set of experiments was performed at isothermal conditions (at constant temperature) at $T=-20^{\circ} \mathrm{C}$, at different constant pressures ranging between 0.1 and 300MPa. Fig.6 schematically shows boundary conditions of both sets of experiments, while the corresponding temperature and the pressure loading profiles for each of the three TPU's is presented in Fig.7. In all experiments, each loading step consists of 3 hours stabilization time and 1000s ( 15min) measuring time. All experiments started at conditions where the material is closer to glassy state i.e., at lowest temperature for the $1^{\text {st }}$ Set and at highest pressure for the $2^{\text {nd }}$ Set of experiments.

In all cases at least three repetitions of measurements were performed, where for each repetition different samples was used. After averaging segments measured at the same boundary conditions, we have applied the Closed Form Shifting (CFS) algorithm, described in Section II C, to create the shear relaxation $G(t)$ mastercurves. The latter were then interconverted into the frequency domain, as described in Section II D, to obtain the storage $G^{\prime}(\omega)$, and the loss $G^{\prime \prime}(\omega)$, moduli for the three examined materials.

Dilatometry measurements were needed for determining constants of the FMT model, described in Section II A. Measurements were again performed with the CMS apparatus using the dilatometer insert, described in Section II B. In this case, the pressure profile was the 
1190A
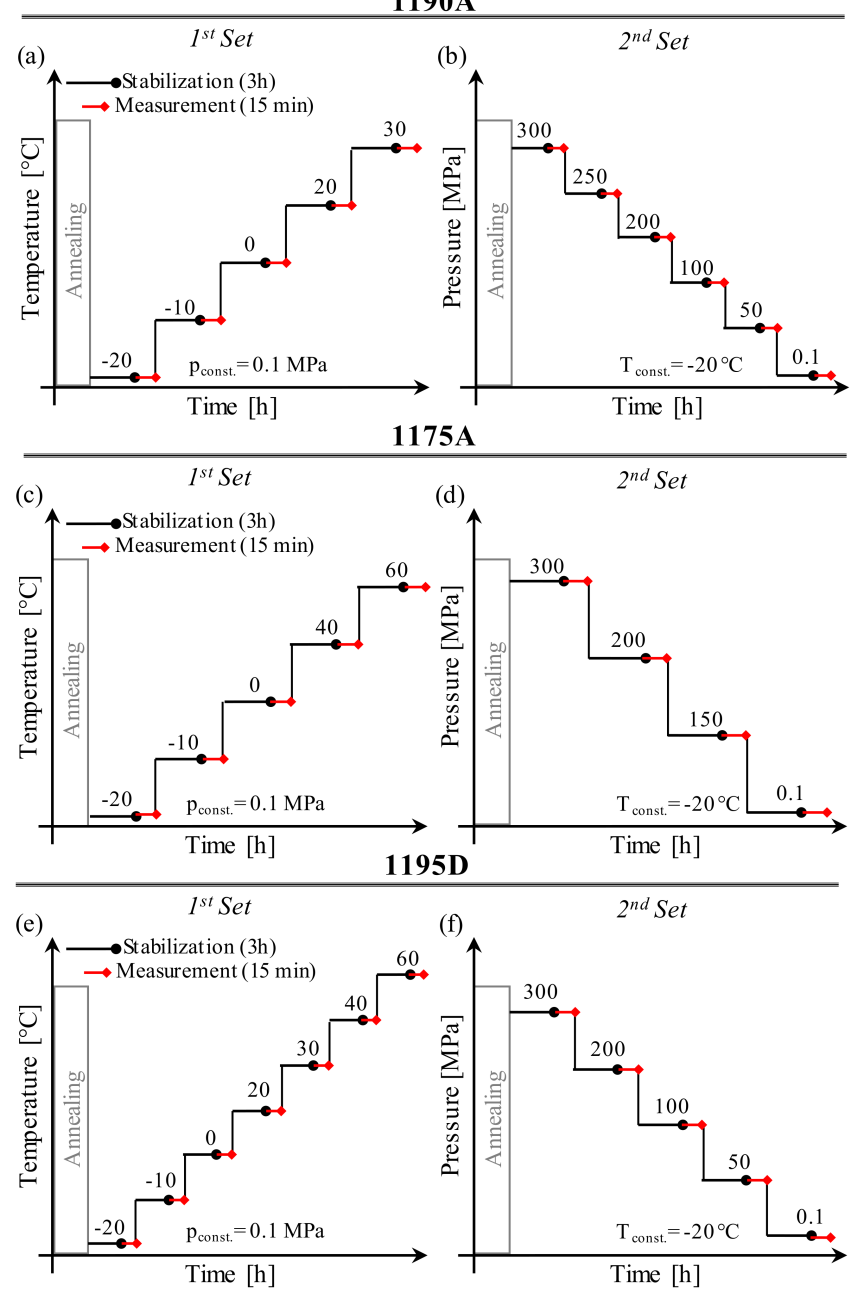

FIG. 7. Temperature/pressure loading profiles for individual materials used in shear relaxation experiments.

same for all three materials, starting at the highest pressure, which was stepwise decreased, as shown in Fig.8. Each loading step consisted of 30min stabilization time followed by $2 \mathrm{~min}$ measuring time of specimen length. For each material, we performed three experiments, always using different fresh samples. After averaging the obtained results, we have calculated the specific volume, using Eq.11, that was needed in calculation of the FMT parameters. The results of the FMT model parameters are displayed in Table IV. 


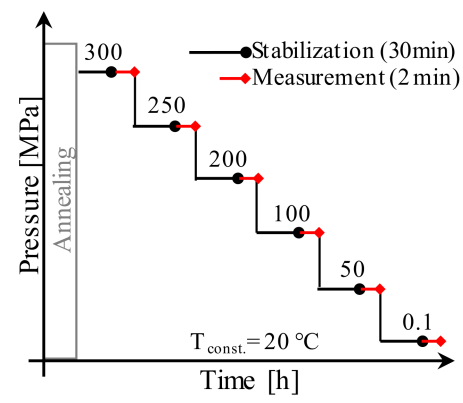

FIG. 8. Pressure loading profile for bulk measurements, performed at $20^{\circ} \mathrm{C}$ for all materials.

\section{RESULTS AND DISCUSSION}

\section{A. Shear relaxation segments and mastercurves}

Following the experimental procedure described in previous section, Section III B, we have performed two sets of shear relaxation experiments on three TPU materials: 1190A, 1175A and 1195D. Both sets of results are shown together in Fig.9(a) - Fig.11(a). The $1^{\text {st }}$ Set of measurements were performed at different temperatures, ranging from about $-20^{\circ} \mathrm{C}$ to $+60^{\circ} \mathrm{C}$, while the pressure was kept constant at $p=0.1 M P a$. These results are shown in Fig.9(a) - Fig.11(a) at the bottom of figures. The $2^{\text {nd }}$ Set of measurements were performed at different pressures, ranging from about 50MPa to $300 \mathrm{MPa}$, at constant temperature at about $T=-20^{\circ} \mathrm{C}$. The results are shown in the same diagrams at the top. The exact, measured temperatures and pressures are indicated in each diagram. The error bars in all diagrams indicate maximal difference between averaged and measured values.

As a next step, we have used the CFS algorithm to construct the three mastercurves at the reference temperature $20^{\circ} \mathrm{C}$ and the reference pressure $0.1 \mathrm{MPa}$, shown in Fig.9(b) - Fig.11(b). It is important to note that combining temperature and pressure boundary conditions allows substantial widening of the experimental time-window in determination of material functions, in our case the relaxation mastercurves. It is evident from Fig.9(b) - Fig.11(b), cf. segments measured at $-20^{\circ} \mathrm{C}$ and $0.1 \mathrm{MPa}$, that performing experiments

just at different temperatures and constant ambient pressure would allow determination of approximately half of the mastercurve only.

For the FMT model the $c_{1}$ and $c_{2}$ constants were obtained through time-temperature superposition using previously described CFS software. Volumetric measurements, described in Section III were used to determine material parameters $K_{e}^{*}$ and $k_{e}$ (and thus $c_{4}$ using Eq.6) 
(a)

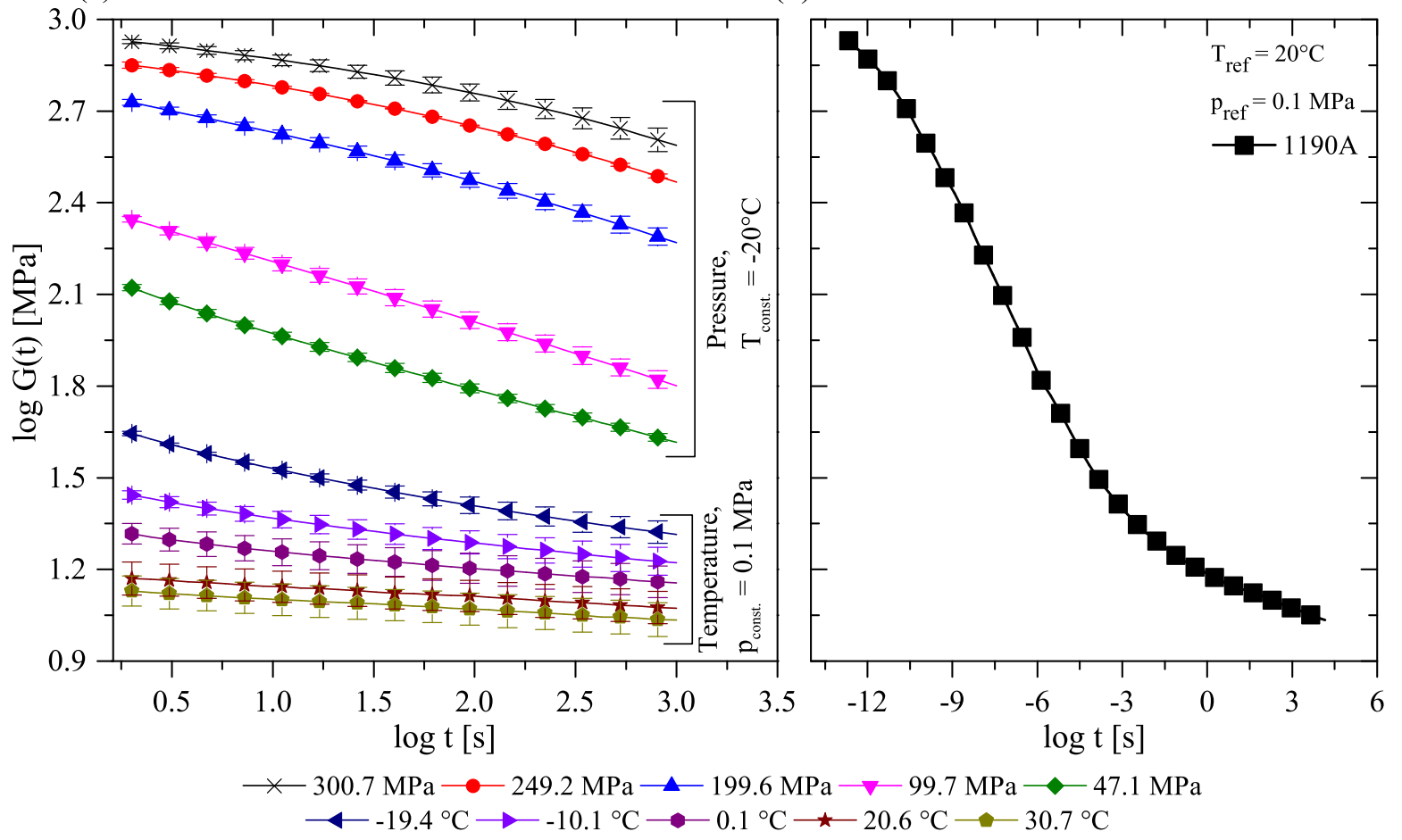

FIG. 9. (a) Segments at different temperature and pressure conditions and (b) mastercurve of bulk material $1190 \mathrm{~A}$ at $T_{r e f}=20^{\circ} \mathrm{C}$ and $p_{r e f}=0.1 \mathrm{MPa}$.

TABLE IV. FMT parameters.

\begin{tabular}{lccccccc}
\hline \hline Mater. & $c_{1}$ & $\begin{array}{c}c_{2} \\
{\left[{ }^{\circ} \mathrm{C}\right]}\end{array}$ & $\begin{array}{c}\alpha_{f} \times 10^{-4} \\
{\left[{ }^{\circ} C^{-1}\right]}\end{array}$ & $\begin{array}{c}K_{e} \\
{[M P a]}\end{array}$ & $k_{e}$ & $\begin{array}{c}K_{\phi} \\
{[M P a]}\end{array}$ & $k_{\phi}$ \\
\hline $1190 \mathrm{~A}$ & 31.24 & 202.43 & 1.26 & 2763 & 12.54 & 3092.74 & 12.59 \\
$1175 \mathrm{~A}$ & 11.46 & 52.35 & 1.87 & 2434 & 12.89 & 2494.02 & 13.97 \\
$1195 \mathrm{D}$ & 24.92 & 105.19 & 1.98 & 2762 & 13.44 & 2877.39 & 15.21 \\
\hline \hline
\end{tabular}

fitting the Eq.9. For the calculation of the remaining constants $c_{3}, c_{5}$ and $c_{6}$, experimental shift factors obtained from measurements at constant temperature $\left(T=-20^{\circ} \mathrm{C}\right)$ and varying pressures, were fitted to Eq.1 using the Levenberg-Marquardt algorithm (MATLAB R2015a). Table IV shows material parameters for all three materials.

Mastercurves for the three materials are compared in double-logarithmic Fig.12(a) and semi-logarithmic Fig.12(b) diagrams. The third figure, Fig.12(c) shows their sensitivity to hydrostatic pressure. From the first, double logarithmic diagram, we observe that the ratio of moduli of the three materials remains fairly constant along the complete time-scale. In this case, the ratio is displayed as a distance between the curves. On the other hand, the 
(a)

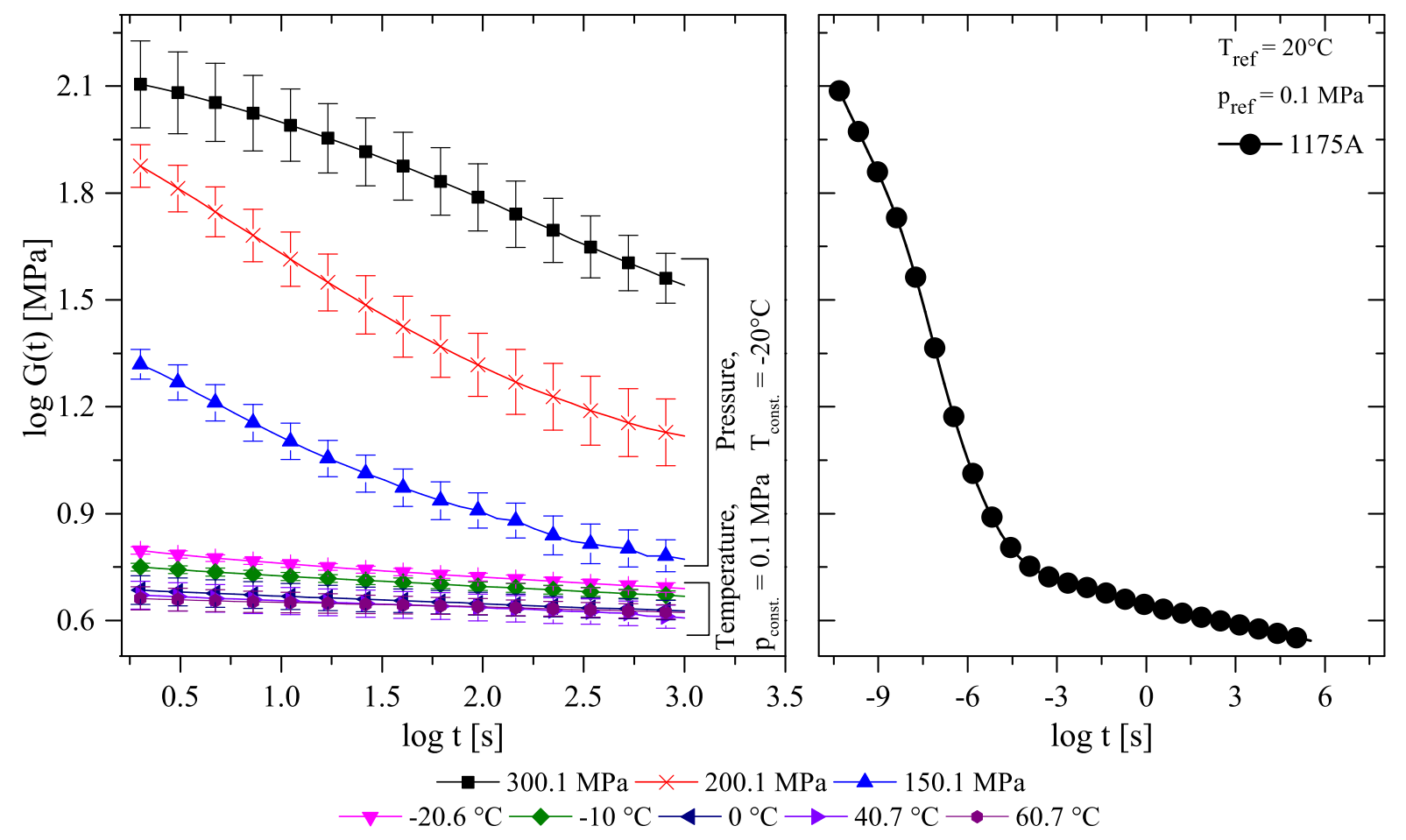

FIG. 10. (a) Segments at different temperature and pressure conditions and (b) mastercurve of bulk material $1175 \mathrm{~A}$ at $T_{r e f}=20^{\circ} \mathrm{C}$ and $p_{\text {ref }}=0.1 \mathrm{MPa}$.

semi-logarithmic diagram allows better comparison of material functions absolute values.

We see that close to the glassy state i.e., at short times, the values of the shear moduli are quite high for 1190A and 1195D and about 6-9 times smaller for 1175A, whereas their moduli become very small and almost indistinguishable at longer times i.e., this is where the materials are used at conditions. Hence, their properties at ambient conditions are for all practical purposes the same. Double logarithmic diagram reveals another interesting feature of the material 1175A, indicated with the two dashed lines in Fig.12(a) i.e., material 1175A exhibits an abrupt change in its behavior at a critical time scale which may be adjusted with pressure and temperature boundary conditions. At this point in time material essentially enters its rubbery state. For the other two TPU materials, this transition is much more gradual.

From Fig.12(c), we see that all three materials are quite sensitive to pressure, the main difference in their pressure sensitivity is visible within the first $100 M P$ a, where 1190A proofs to be most sensitive of the three materials. At the highest pressure (300MP $a)$, properties of 1190A are shifted for around 5.5 decades along the logarithmic time-, and/or 
(a)

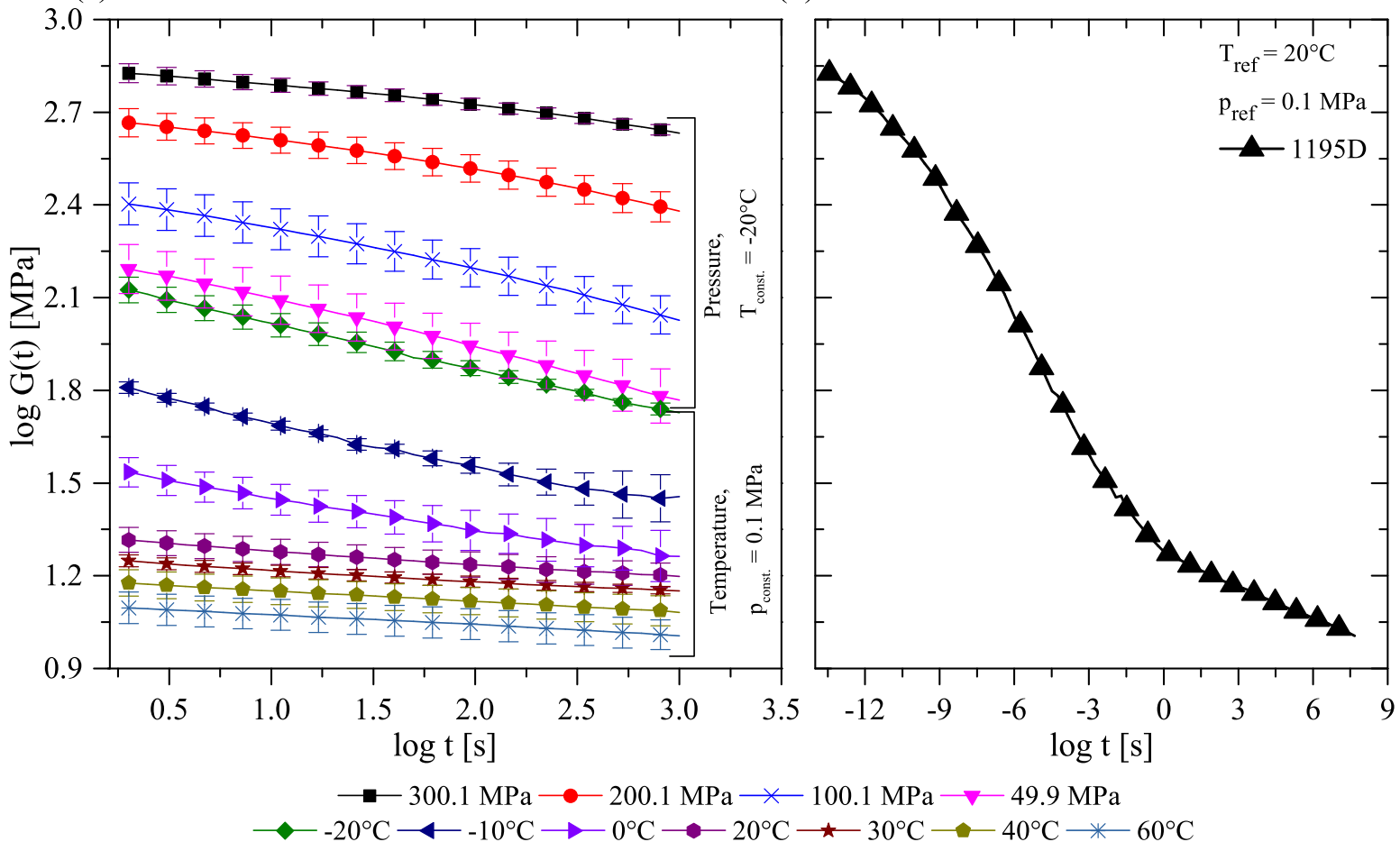

FIG. 11. (a) Segments at different temperature and pressure conditions and (b) mastercurve of bulk material $1195 \mathrm{D}$ at $T_{r e f}=20^{\circ} \mathrm{C}$ and $p_{\text {ref }}=0.1 \mathrm{MPa}$.

(a)

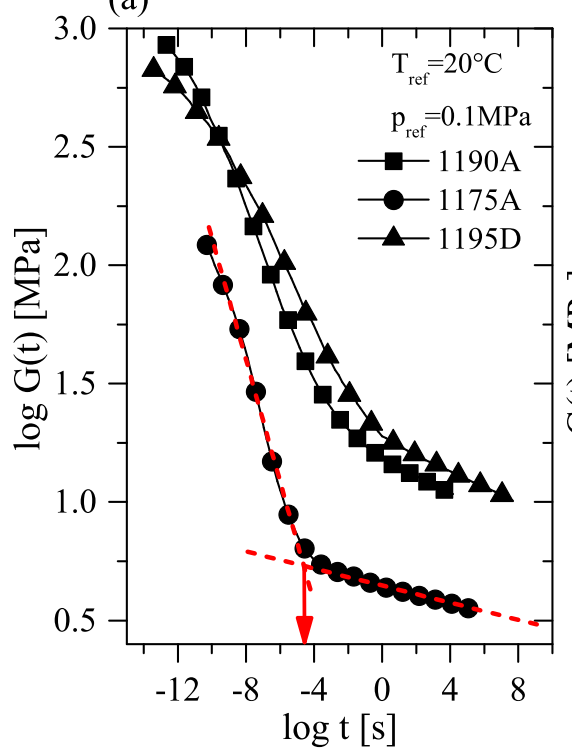

(b)

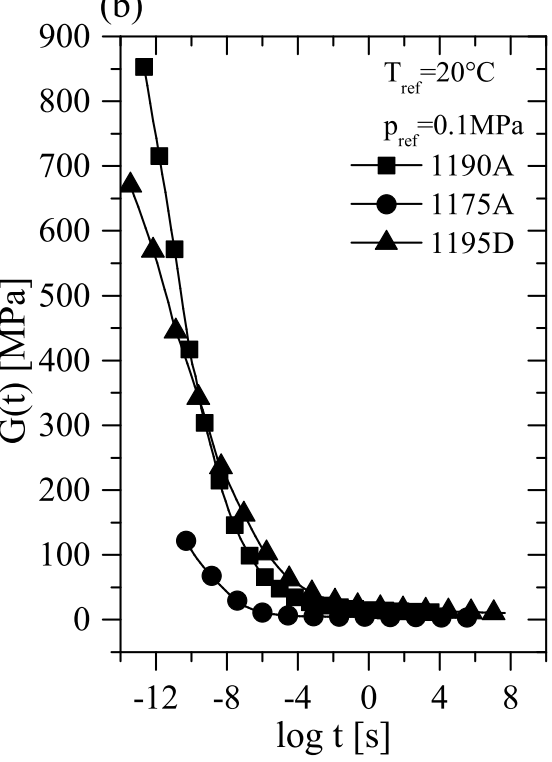

(c)

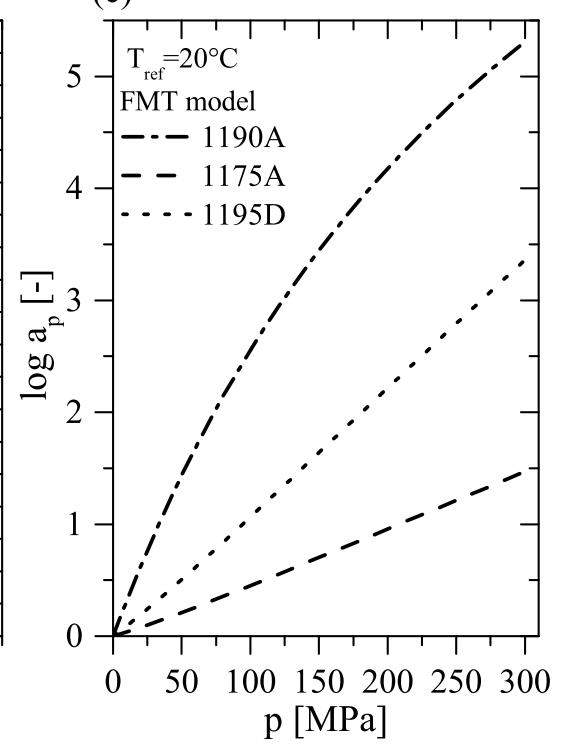

FIG. 12. Mastercurves at $T_{r e f}=20^{\circ} \mathrm{C}$ and $p_{\text {ref }}=0.1 \mathrm{MPa}$ in (a) double-logarithmic, and (b) semi-logarithmic coordinate system, and (c) pressure shift factors $\log a_{p}(p)$ of three bulk TPU materials. 
frequency scale. For 1195D, this shift is about 3.5, whereas for $1175 \mathrm{~A}$ properties are shifted along the logarithmic time/frequency-scale for about 1.5 decades only.

\section{B. Dynamic material functions}

Using the approximate interrelations Eq.28 and Eq.29, we have calculated the storage $G^{\prime}(\omega)$, and the loss $G^{\prime \prime}(\omega)$, moduli for the three examined materials. The results are shown in Fig.13, again in double logarithmic and semi-logarithmic coordinate systems. Figs.13(a) and (c) show the storage modulus $G^{\prime}(\omega)$, whereas Figs.13(b) and (d) the loss modulus $G^{\prime \prime}(\omega)$. The difference between materials in time domain (Fig.12) is also reflected in the frequency domain.

The storage modulus $G^{\prime}(\omega)$, which defines the vibro-isolation stiffness, increases with excitation frequency for about 100 times for 1190A and 1195D, and for about 10 times for 1175A. However, as seen from the semi-logarithmic diagrams, within the frequency range indicated as shaded area, which is of interest for engineering applications, their stiffness is quite low. In the case of $1175 \mathrm{~A}$ we may see that it behaves elastically i.e., its storage modulus is almost constant (up to $10^{4} \mathrm{~Hz}$ ), whereas, within the same frequency range, the stiffness of other two materials exhibit quite strong frequency dependence. From the double logarithmic diagram, we see that $1175 \mathrm{~A}$ exhibits an abrupt transition at $10^{4} \mathrm{~Hz}$, while for the other two materials the transition is more gradual.

The loss modulus $G^{\prime \prime}(\omega)$, also increases with excitation frequency for all three materials, again for about 100 times for 1190A and 1195D, and for about 10 times for 1175A. Since the loss modulus $G^{\prime \prime}(\omega)$, defines the damping ability of a vibro-isolator, the higher values mean better damping. However, from the Figs.13(b) and (d) we may see that the maximal measured values in all three cases are located at very high frequencies, between $10^{8}-10^{12} \mathrm{~Hz}$. In addition to this, it may also be seen that the values of the loss modulus $G^{\prime \prime}(\omega)$, in the frequency range of interest in most engineering applications, are relatively low i.e. in the range between 0.1 to $25 \mathrm{MPa}$, which is not bad in comparison to metals, however far away from real potentials of TPU materials.

Comparing the three TPUs shows that the material 1175A has the lowest loss modulus $G^{\prime \prime}(\omega)$ through the whole frequency range. As in the case of storage modulus $G^{\prime}(\omega), 1175 \mathrm{~A}$ abruptly enters the transition state at about $10^{3} \mathrm{~Hz}$ where its energy absorption properties 
(a)
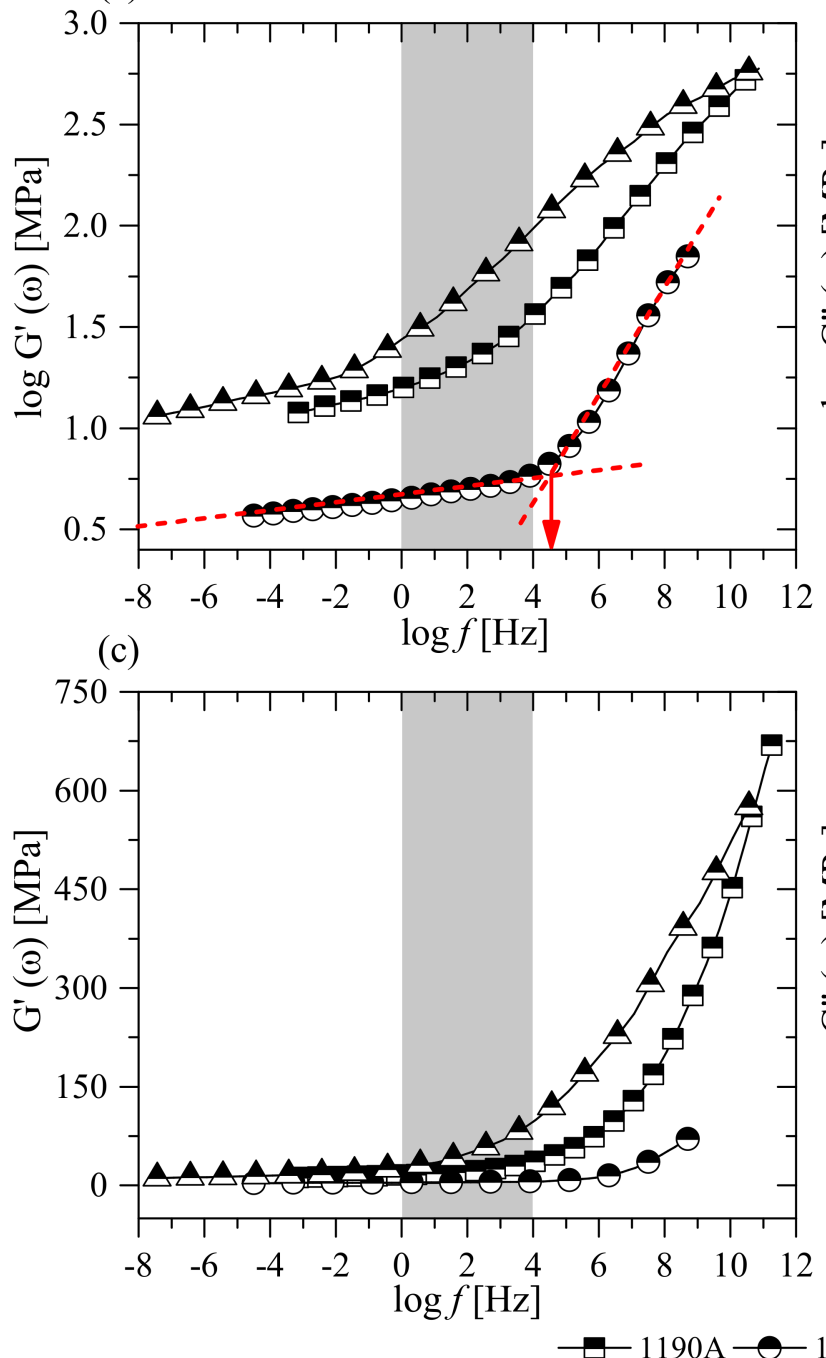

(b)
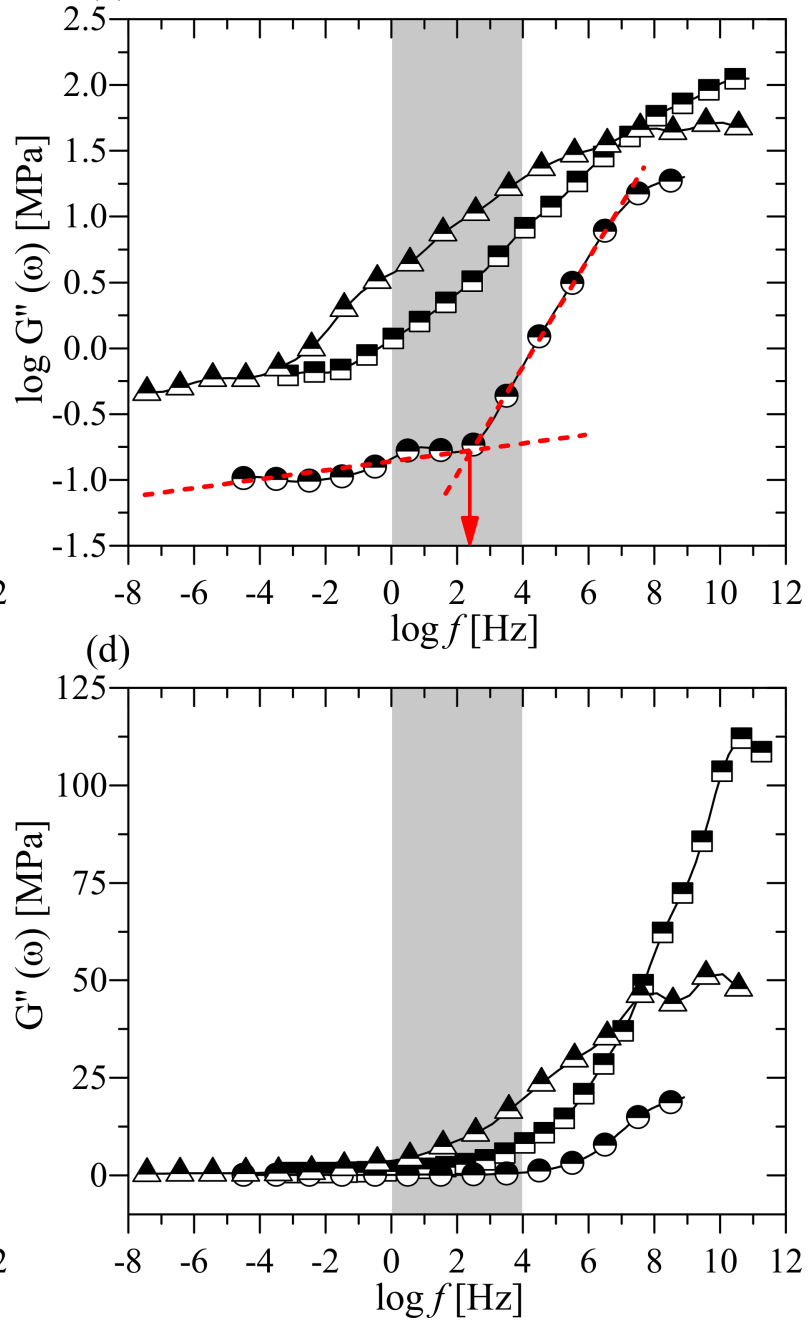

FIG. 13. Shear storage modulus $G^{\prime}(\omega)$ in (a) double logarithmic and (b) semi-logarithmic coordinate systems; and shear loss modulus $G^{\prime \prime}(\omega)$ in (c) double logarithmic and (d) semi-logarithmic coordinate systems. All figures are shown for bulk materials at $T_{r e f}=20^{\circ} \mathrm{C}$ and $p_{\text {ref }}=0.1 \mathrm{MPa}$.

are strongly excitation frequency dependent, before that its dissipation properties are more or less constant, see Fig.13(b). The transition is much more gradual for 1190A and 1195D.

\section{Analysis of GDE potential}

Combining the information on dynamic viscoelastic material functions presented in Figs.13 with materials hydrostatic pressure sensitivity, presented in Fig.12, we may construct 3D diagrams showing interrelation between the storage $G^{\prime}(\omega)$, and the loss $G^{\prime \prime}(\omega)$, modulus, and excitation frequency and hydrostatic pressure to which material is exposed. 

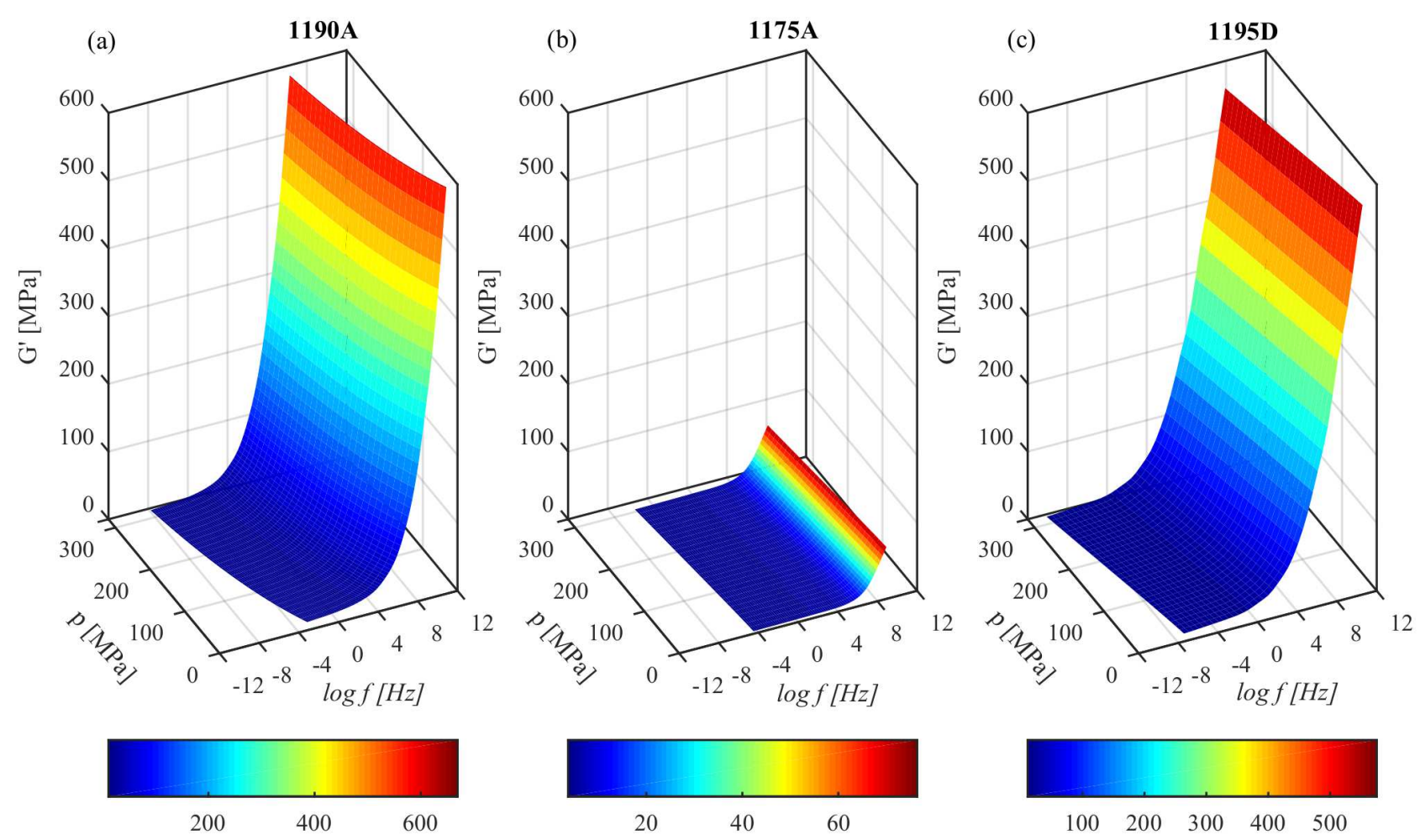

FIG. 14. Frequency dependent shear storage modulus $G^{\prime}(\omega)$ in dependence of hydrostatic pressure for (a) $1190 \mathrm{~A}$, (b) $1175 \mathrm{~A}$ and (c) $1195 \mathrm{D}$ at $T_{r e f}=20^{\circ} \mathrm{C}$.

The results are shown in Fig.14 for the storage modulus $G^{\prime}(\omega)$, and in Fig. 15 for the loss modulus $G^{\prime \prime}(\omega)$.

The 3D diagrams provide a general inside how the two material functions depend on hydrostatic pressure and excitation frequency. The differences between the three materials are obvious, particularly when comparing their loss moduli. However, for more precise analysis of how hydrostatic pressure affects the storage $G^{\prime}(\omega)$ and loss $G^{\prime \prime}(\omega)$, moduli within the vibro-isolation range $1-10000 \mathrm{~Hz}$ that is of interest, we need to perform some further analysis. Specifically, we are interested how much one can increase the storage $G^{\prime}(\omega)$ modulus (related to stiffness) and loss $G^{\prime \prime}(\omega)$ modulus (related to energy absorption) of a vibroisolation by exposing the material to selected hydrostatic pressure.

Stiffness of an vibro-isolator depends on its geometry and the storage modulus of a material from which it is built, whereas, its energy absorption capability is defined by the isolator total volume and material loss modulus. Hence, if we keep the geometry and volume of an isolation constant, its stiffness and its energy absorption capability will depend on material storage and loss modulus only. In fact, our main interest is to understand to what 

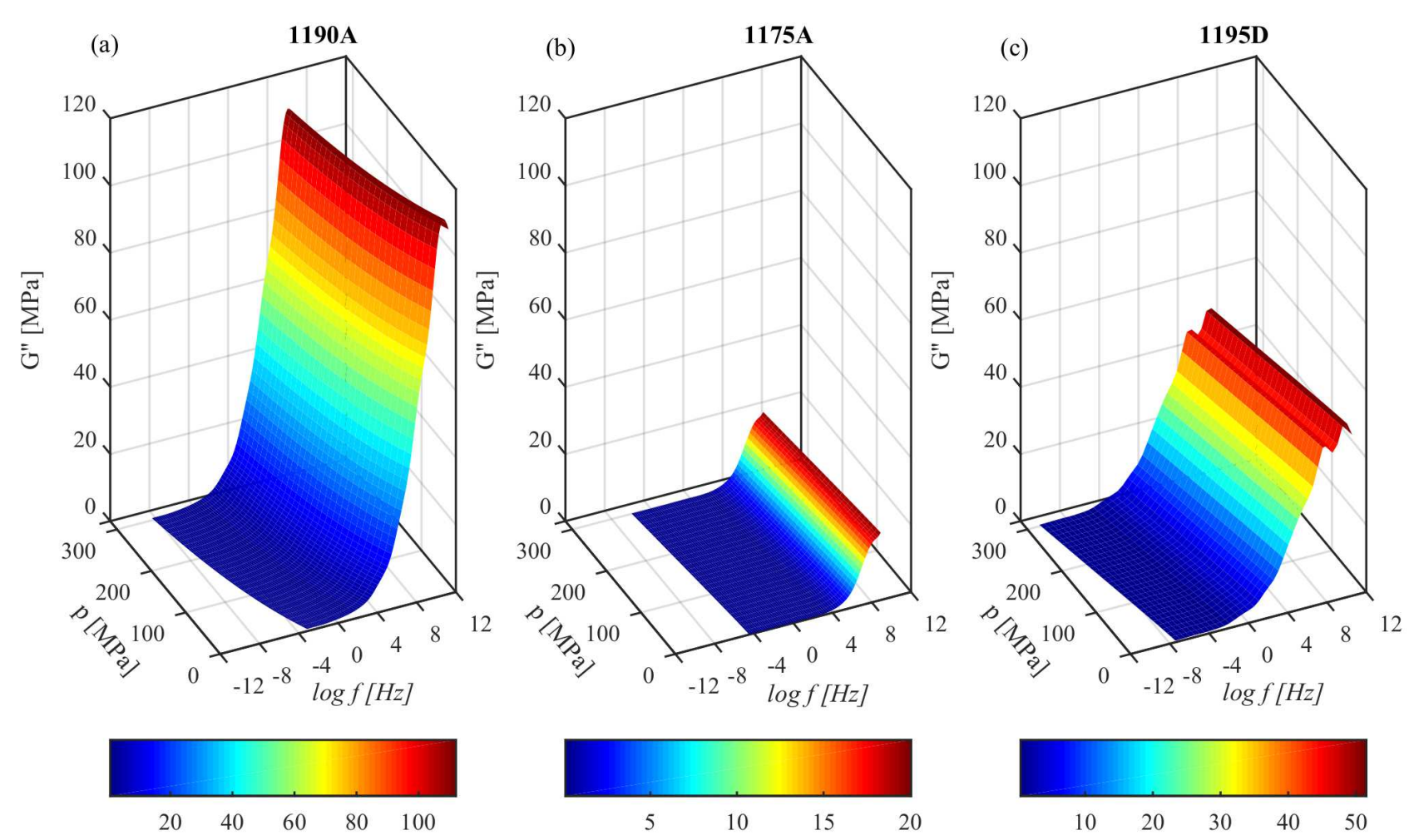

FIG. 15. Frequency dependent shear loss modulus $G^{\prime \prime}(\omega)$ in dependence of hydrostatic pressure for (a) 1190A, (b) $1175 \mathrm{~A}$ and (c) $1195 \mathrm{D}$ at $T_{r e f}=20^{\circ} \mathrm{C}$.

extent (how many times) we can increase stiffness and energy absorption of an isolation by exposing the material from which it is made of to a selected hydrostatic pressure. Therefore, we introduce two parameters, first, defining the increase of vibro-isolator stiffness by exposing it to a selected hydrostatic pressure $p$, relative to the vibro-isolator stiffness at the ambient pressure $p_{0}=0.1 M P a$ :

$$
\kappa_{k}(p, \omega)=\frac{G^{\prime}(p, \omega)}{G^{\prime}\left(p_{0}, \omega\right)},
$$

and the second

$$
\kappa_{d}(p, \omega)=\frac{G^{\prime \prime}(p, \omega)}{G^{\prime \prime}\left(p_{0}, \omega\right)}
$$

which defines an increase of isolation energy absorption (damping) obtained by exposing the analyzed TPUs to a selected hydrostatic pressure.

All comparisons are made within the frequency range $(1-10000 \mathrm{~Hz})$, which is of interest for impact and vibration isolation. The results are shown in two different forms: (i) $\kappa_{k}(p, \omega)$ and $\kappa_{d}(p, \omega)$ as functions of excitation frequency, for four selected pressures; and (ii) $\kappa_{k}(p, \omega)$ and $\kappa_{d}(p, \omega)$ as functions of hydrostatic pressure, for four selected excitation 
frequencies. In fact, both mentioned diagrams present the same information i.e., how much hydrostatic pressure we need at a given excitation frequency to obtain a desired increase of isolation energy absorption and/or stiffness. However, they present this information from a different point of views which is important for designing an vibro-isolator for a specific engineering application. In our discussion in Section IV B we have pointed out that energy absorption and stiffness of an vibro-isolator made from any of the three materials are within the excitation frequency range of interest i.e., $1-10^{4} \mathrm{~Hz}$ approximately constant. As demonstrated in continuation, this is not necessarily so when we use hydrostatic pressure to modify vibro-isolator characteristics. In this case, both, stiffness and energy absorption characteristics of an vibro-isolator may become (strongly) excitation frequency dependent.

\section{1. $\kappa_{k}(p, \omega)$ and $\kappa_{d}(p, \omega)$ as functions of frequency}

Figs.16 and 17 show isolation excitation-frequency dependence, which stiffness and energy absorption characteristics were modified by exposing materials to selected hydrostatic pressures i.e., 50, 100, 200 and 300MPa. In Fig.16 we first show frequency dependence of the ratio $\kappa_{k}(p$,$) for (a) 1190A, (b) 1175A, and (c) 1195D, for the four selected pressures,$ which demonstrates improvement of isolation stiffness.

Comparing the three diagrams we clearly see the significant difference between the three materials in the effect of pressure on improvement of isolation stiffness. Within the frequency range of interest, TPU 1190A is very sensitive to pressure and excitation frequency to which the material (isolation) is exposed. Depending on the excitation frequency its stiffness may be increased between 3.5 and 9.5 times by exposing it to hydrostatic pressure of $300 \mathrm{MPa}$. Hence, TPU 1190A is a good material for the new generation GDE damping elements, though its stiffness will be quite sensitive to excitation frequency.

This paper is not the place to discuss this in detail, but let us just mention that this feature may be cleverly utilized in vibration isolation solutions. For example, if a mechanical system with one of the currently existing isolations operates below the resonance frequency, an increase of excitation frequency will bring it closer to (or even into) its resonance, possibly causing its failure. However, if one uses GDE vibro-isolator made of TPU 1190A instead an increase of excitation frequency will cause an increase of isolation stiffness, as indicated with two arrows in Fig.16(a). As a result, the resonance frequency of such mechanical 
(a)

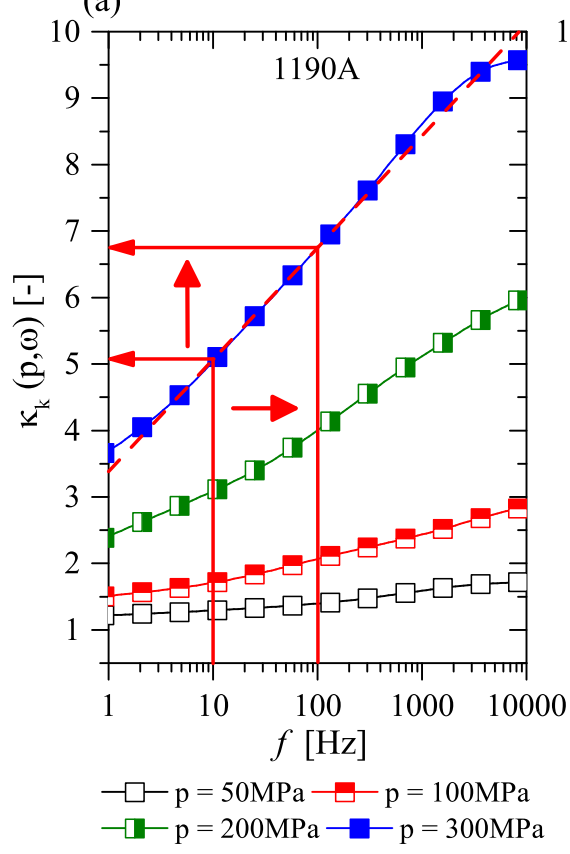

(b)

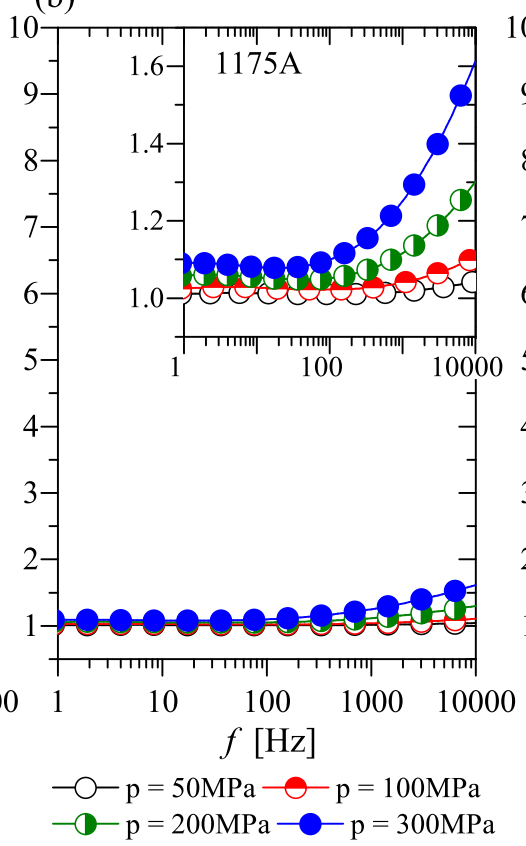

(c)

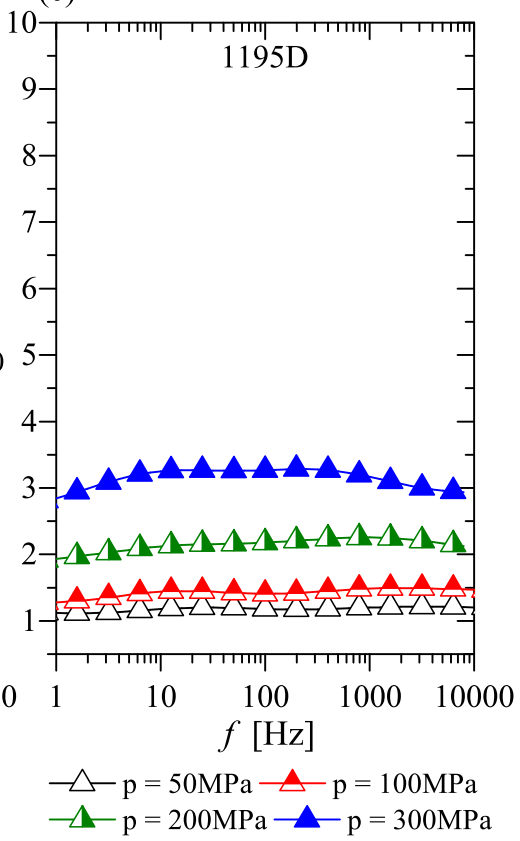

FIG. 16. $\kappa_{k}(p, \omega)$ as functions of frequency for four selected pressures and materials (a) 1190A, (b) $1175 \mathrm{~A}$ and (c) 1195D. Arrows in (a) indicating an increase of isolation stiffness with excitation frequency in selected frequency range.

system will be automatically moved to higher frequencies, hence, the system will not enter its resonance. In fact, GDE vibro-isolator allows building mechanical systems that in real engineering situations will newer experience problems related to resonance.

Material TPU 1175A, Fig.16(b), in comparison to 1190A, exhibits diametric properties, stiffness of an isolation made from such material will be up to $300 M P a$ pressure and excitation frequency (almost) independent. Hence, one cannot utilize hydrostatic pressure to improve/modify stiffness of isolations made from TPU 1175A. Finally, TPU 1195D based isolation will exhibit characteristics that are combination of the two previous materials, Fig.16(c). Its stiffness is pressure dependent, not as strongly as 1190A, however, it remains practically constant within the complete excitation-frequency range of interest. With a pressure of $300 \mathrm{MPa}$ one may increase the stiffness of an isolation for about 3 times.

Let us now turn to isolation energy absorption properties, displayed in Fig.17, which shows $\kappa_{d}(p, \omega)$ as function of excitation frequency for the four selected hydrostatic pressures i.e., 50, 100, 200 and 300MPa. The results for the three materials are shown in the same order as before. Comparing the three diagrams we again observe strong difference between the three materials, however, the effect of pressure is in this case even stronger and different 
(a)

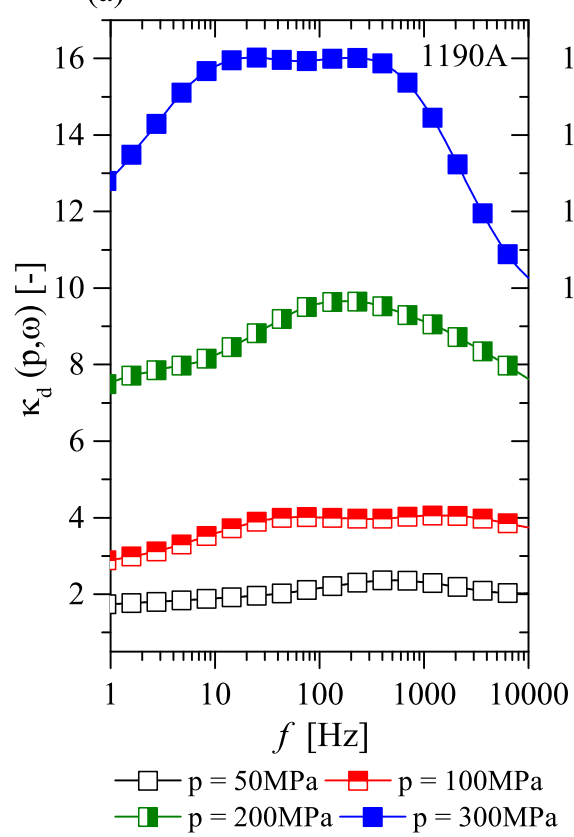

(b)

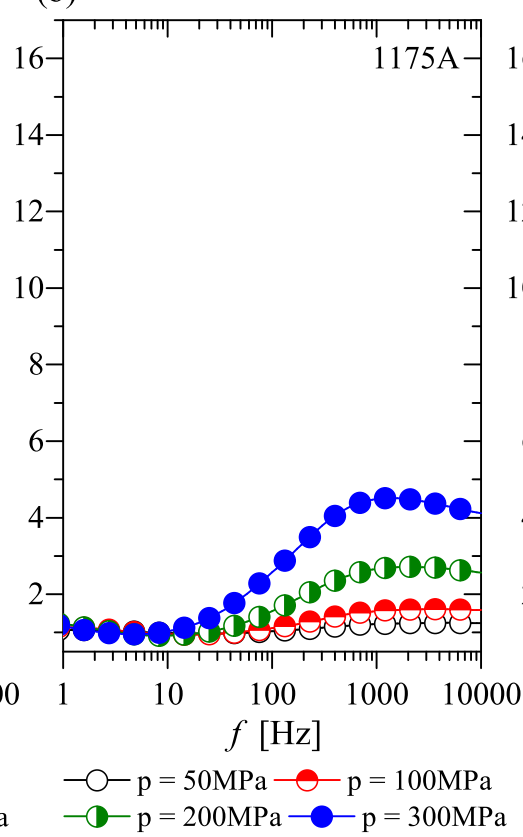

(c)

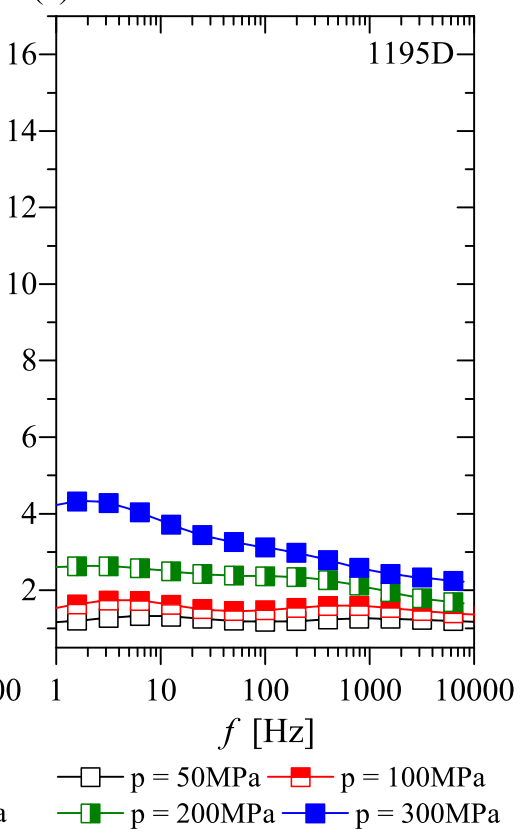

FIG. 17. $\kappa_{d}(p, \omega)$ as functions of frequency for four selected pressures and materials (a) 1190A, (b) $1175 \mathrm{~A}$ and (c) 1195D.

than in the case of isolator stiffness. In case of 1190A, Fig.17(a), when exposed to 300MPa, the improvement of its energy absorption capability is about 16 times. In addition, within the range $10 \mathrm{~Hz}$ to $1000 \mathrm{~Hz}$ material energy-absorption is excitation-frequency independent. In fact, at $100 \mathrm{MPa}$ one observes an improvement of 4 times, for excitation-frequencies above $20 \mathrm{~Hz}$, whereas, when material is exposed to $50 \mathrm{MPa}$ one observes improvement of two times within the complete frequency range of interest. High energy absorption and an increase of isolation stiffness caused by excitation frequency makes TPU 1190A a very attractive material for the new generation GDE damping elements. The two characteristics work hand-in-hand, high energy absorption diminishes vibrations per se, in addition an increase of isolation stiffness will move away mechanical system resonance frequency causing additional reduction of vibrations.

Material 1175A, Fig.17(b), which its stiffness was pressure independent (Fig.16(b)), exhibits sensitivity to pressure within the complete frequency range. It is interesting that up to $30 \mathrm{~Hz}$ an improvement of about 1.5 times is observed at all four selected pressures, however, at higher excitation-frequencies $1175 \mathrm{~A}$ becomes more sensitive to pressure. At $300 \mathrm{MPa}$ the energy absorption capability may increase more than 4 times, however the improvement is observed only at excitation-frequencies larger than $100 \mathrm{~Hz}$. Hence, we may conclude that 
1175A should be used when excitation-frequencies are larger than $100 \mathrm{~Hz}$.

Material 1195D, Fig.17(c), at pressures up to 100MPa exhibits similar energy absorption properties as $1175 \mathrm{~A}$ i.e., its performance improvement is about 1.5 times, and it is approximately constant throughout the frequency range of interest. In contrast to $1175 \mathrm{~A}, 1195 \mathrm{D}$ exhibits, at pressures above 200MPa, the largest increase of energy absorption capability at low excitation frequencies. The improvement of energy absorption at $300 \mathrm{MPa}$ increases up to 4 times.

One may conclude that energy absorption properties of 1175A and 1195D (within the frequency range of interest) mirror each other, TPU 1175A exhibits high pressure induced energy absorption at high excitation-frequencies, whereas TPU 1195D does so at low frequencies. Hence, an isolation made of two types of materials, one from 1175A and the other from 1195A, will exhibit high energy absorption through the complete frequency range of interest.

\section{2. $\kappa_{k}(p, \omega)$ and $\kappa_{d}(p, \omega)$ as functions of hydrostatic pressure}

In this section we analyze the three materials from different perspective, we examine pressure dependence of isolation stiffness, represented by $\kappa_{k}(p, \omega)$, shown in Fig.18, and of isolation energy absorption, represented by $\kappa_{d}(p, \omega)$, shown in Fig.19, at four selected excitation frequencies i.e., 1, 100, 1000 and $10000 \mathrm{~Hz}$.

We will again start with analyzing pressure induced increase of vibro-isolator stiffness. Fig.18(a) shows $\kappa_{k}(p, \omega)$ for material 1190A at four selected frequencies, Fig.18(b) shows $\kappa_{k}(p, \omega)$ for material $1175 \mathrm{~A}$, where the results are also shown in a close-up diagram in the upper right corner, and Fig.18(c) shows $\kappa_{k}(p, \omega)$ for material 1195D.

The three diagrams in Fig.18 show essentially the same information as that in Fig.16, however, this time $\kappa_{k}(p, \omega)$ is presented as function of pressure to which materials are exposed. We see that for all three materials pressure sensitivity represented with the slope $\lambda_{k}(p, \omega)=\left(\mathrm{d} \kappa_{k}(p, \omega)\right) / \mathrm{d} p$, increases with pressure and excitation frequency. This is most pronounced for 1190A, where for excitation frequency of $1 H z \lambda_{k}(p>100 M P a, \omega=1 H z) \cong$ 1, and for $10^{4} \mathrm{~Hz} \lambda_{k}\left(p>100 M P a, \omega=10^{4} \mathrm{~Hz}\right) \cong 3.5 /(100 M P a)$. As we have already discussed, material $1175 \mathrm{~A}$ is within the frequency range of interest practically pressure independent i.e., $\lambda_{k}(p, \omega) \approx 0$, except for frequencies close to $10000 \mathrm{~Hz}$. Whereas, material 
(a)

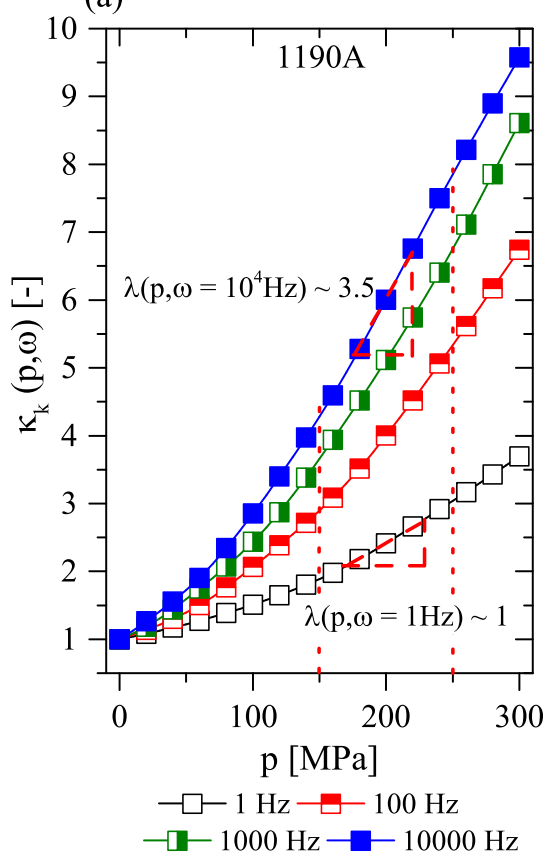

(b)

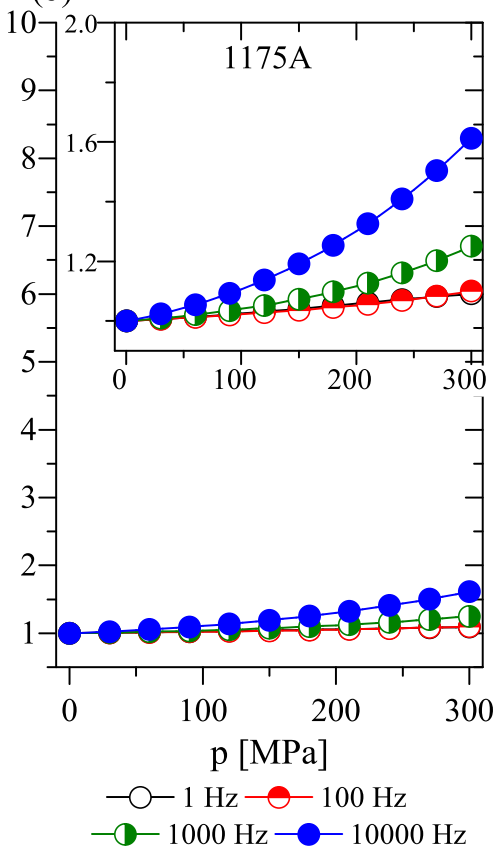

(c)

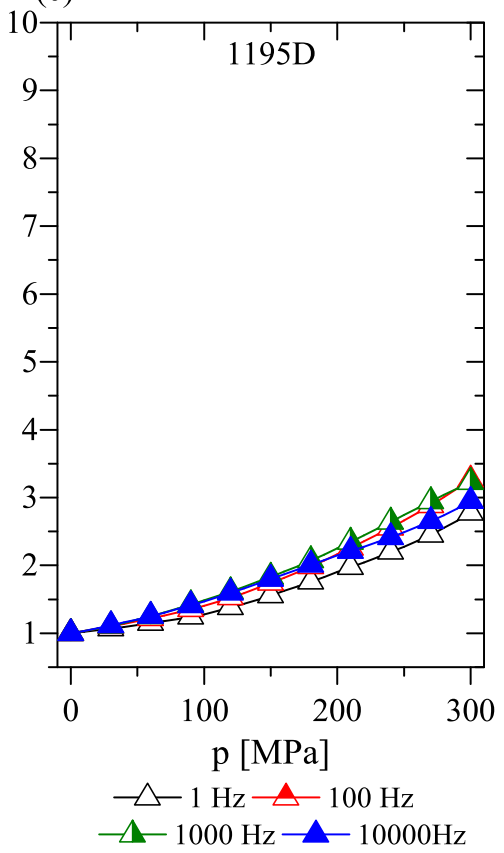

FIG. 18. $\kappa_{k}(p, \omega)$ as functions of hydrostatic pressure at selected frequencies for (a) 1190A, (b) $1175 \mathrm{~A}$ and $(\mathrm{c}) 1195 \mathrm{D}$.

1195D exhibits approximately the same pressure sensitivity for all excitation frequencies i.e., $\lambda_{k}(p, \omega)=0.6 /(100 M P a)$. Hence, regarding the modification of DGE isolation stiffness TPU 1190A appears to be the best material, and to a certain degree 1195D, where one can achieve improvements up to 3 times.

Capability of improving energy absorption with hydrostatic pressure, $\kappa_{d}(p, \omega)$, for the three materials is shown in Fig.19, again for four selected excitation frequencies i.e., 1, 100, 1000 and $10000 \mathrm{~Hz}$. Materials pressure sensitivity may be again represented as $\lambda_{d}(p, \omega)=$ $\left(\mathrm{d} \lambda_{d}(p, \omega)\right) / \mathrm{d} p$ i.e., with the slope of $\kappa_{d}(p, \omega)$. From Fig.19 we observe that energy absorption of all three materials is pressure dependent and it varies with excitation frequency.

The strongest pressure dependence is found with 1190A, where the improvement can be as high as 16 times, whereas the other two materials, 1175A and 1195D, are to a large extent similar, for both the improvement is about 4 times. However, their frequency dependence is quite different, this is particularly true for $1175 \mathrm{~A}$ and $1195 \mathrm{D}$, as we have already discussed in previous section. 
(a)

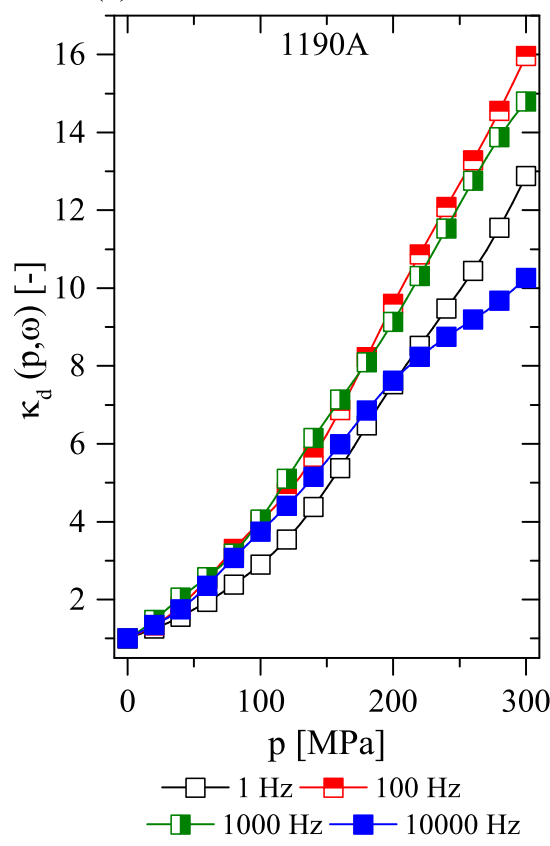

(b)

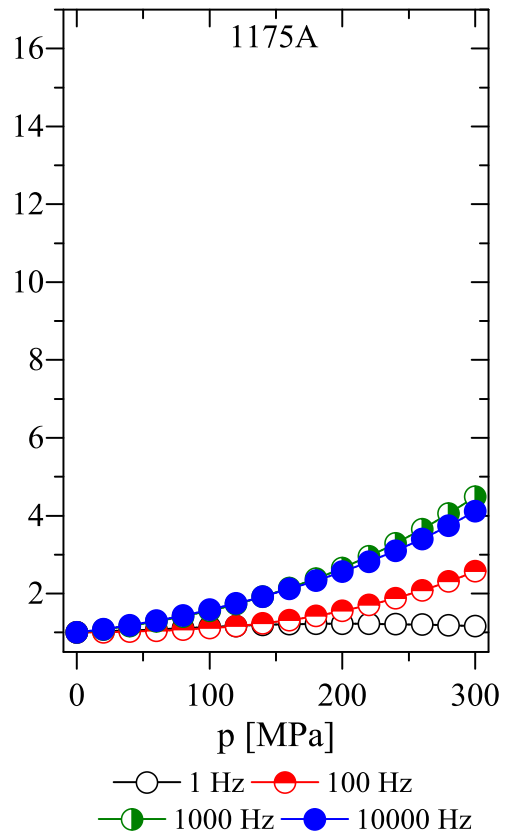

(c)

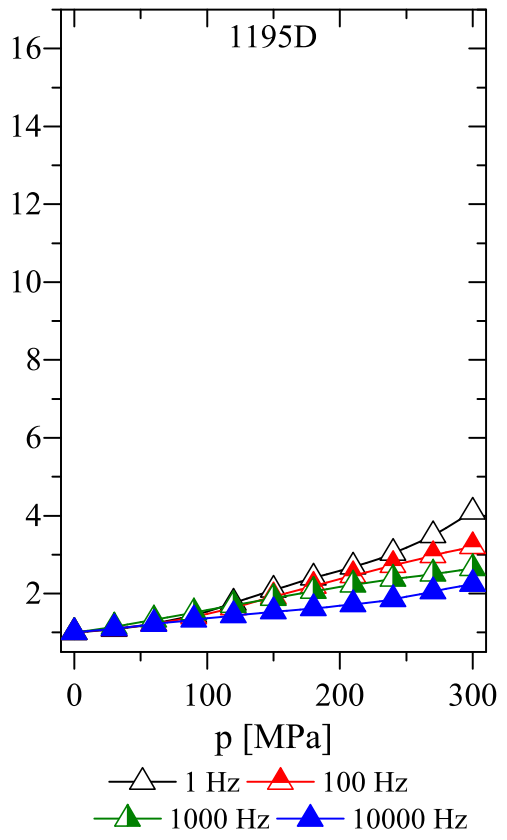

FIG. 19. $\kappa_{d}(p, \omega)$ as functions of hydrostatic pressure at selected frequencies for (a) 1190A, (b) $1175 \mathrm{~A}$ and (c) 1195D.

\section{SUMMARY AND CONCLUSIONS}

This paper presents an analysis of the pressure dependence of three BASF Elastollan ${ }^{\circledR}$ materials i.e., (i) 1190A, (ii) 1175A, and (iii) 1195D in time and frequency domain, that are used in manufacturing of isolation systems. The aim of the characterization was to analyze how much one can enhance performance of an isolation by using these materials in Granular damping elements (GDE), that are based on a patented Dissipative bulk and granular systems technology. DGE isolation uses polymeric materials in granular form to enhance their dynamic properties by exposing them to hydrostatic pressure, which shifts material energy absorptions maxima towards lower frequencies, to match the excitation frequency of dynamic loading to which a mechanical system is exposed.

Pressure dependence of the three materials were characterized in time domain using a self-built high-pressure apparatus for shear-relaxation and volumetric measurements. All samples were prepared under good care and annealed to avoid effects of physical aging. Relaxation experiments were performed in isobaric state (at constant pressure) at several different constant temperatures, and in isothermal state (at constant temperature) at several different constant pressures. Following the time-temperature-pressure superposition 
principle the measured segments where then shifted along the logarithmic time-scale, using the close-from-shifting (CFS) algorithm, to obtain the unique, person-independent, mastercurves, shown in Figs.9-11. Time-temperature-pressure superposition was modeled with the FMT (Fillers-Moonan-Tschoegl) model, for which additional volumetric compressibility measurements were performed. For the three materials, the parameters of FMT model are given in Table IV.

Frequency dependent material functions i.e., shear storage $G^{\prime}(\omega)$ (related to isolation stiffness) and loss modulus $G^{\prime \prime}(\omega)$ (related to isolation energy absorption), were calculated through interconversion, using an approximate method proposed by Schwarzl. The obtained frequency domain material functions, $G^{\prime}(\omega)$ and $G^{\prime \prime}(\omega)$, for the three materials are shown in Figs.13-15.

To study and understand to what extent (how many times) one can increase stiffness and energy absorption of a vibro-isolators we have introduced two parameters i.e., $\kappa_{k}(p, \omega)$ and $\kappa_{d}(p, \omega)$. The first defines the increase of stiffness, and the second the increase of energy absorption. These comparisons were made within the frequency range $(1-10000 H z)$, which is of interest for impact and vibration isolation.

From the obtained results one may draw the following conclusions:

1. The three polyether-based thermoplastic polyurethane (TPU) materials from the Elastollan ${ }^{\circledR} 11$ series i.e., (i) $1190 \mathrm{~A}$, (ii) $1175 \mathrm{~A}$, and (iii) $1195 \mathrm{D}$, with similar chemical structure, exhibit significantly different time- and frequency-dependent properties.

2. In time domain, the strength of the shear moduli of $1190 \mathrm{~A}$ and $1195 \mathrm{D}$ is about $6-9$ times higher than that of $1175 \mathrm{~A}$, whereas, their moduli become very small and almost indistinguishable at longer times i.e., their properties at ambient conditions are for all practical purposes the same.

3. All three materials are quite sensitive to pressure, and $1190 \mathrm{~A}$ proofs to be the most sensitive of the three materials. At $300 \mathrm{MPa}$, properties of $1190 \mathrm{~A}$ are shifted along the logarithmic time-, and/or frequency scale for around 5.5 decades, for 1195D and 1175A this shift is only about 3.5 and 1.5 decades, respectively. These shifts may be achieved by exposing materials to selected hydrostatic pressure.

4. The storage modulus $G^{\prime}(\omega)$, which defines the vibro-isolation stiffness within the fre- 
quency range of interest, may be increased with pressure for about 100 times for 1190A and $1195 \mathrm{D}$, and for about 10 times for 1175A.

5. The loss modulus $G^{\prime \prime}(\omega)$, which defines the vibro-isolation energy absorption capability within the frequency range of interest, may be increased with pressure for again about 100 times for 1190A and 1195D, and for about 10 times for 1175A.

In conclusion, among the three measured materials it was shown that material 1190A seems to be the most promising for the use in Granular damping elements. Besides having the largest values of loss modulus $G^{\prime \prime}(\omega)$ it is also the most sensitive to pressure. Meaning, that even at relatively small applied hydrostatic pressures the increase of loss modulus $G^{\prime \prime}(\omega)$ is higher compared to other two materials.

\section{ACKNOWLEDGMENTS}

The authors wish to thank Uroš Mavec and Ernest Tripodi for their contribution in performing shear relaxation measurements, and BASF for their generous donation of TPU materials. We also want to acknowledge the partial financial support of the Slovenian Research Founding Agency, under the contract P2-0264.

\section{REFERENCES}

${ }^{1} \mathrm{C}$. Hansen, Effects of low-frequency noise and vibration on people (Brentwood, UK, 2007).

${ }^{2}$ M. J. Crocker, Handbook of noise and vibration control (John Wiley \& Sons, 2007).

${ }^{3}$ D. D. L. Chung, "Review: Materials for vibration damping," Journal of Materials Science 36, 5733-5737 (2001).

${ }^{4}$ E. I. Rivin, Passive vibration isolation (Asme press New York, 2003).

${ }^{5}$ R. Lakes, Viscoelastic Materials (Cambridge University Press, 2009).

${ }^{6}$ D. I. G. Jones, Handbook of viscoelastic vibration damping (John Wiley \& Sons, 2001).

${ }^{7}$ R. S. Lakes, "High Damping Composite Materials: Effect of Structural Hierarchy," Journal of Composite Materials 36 (2002).

${ }^{8}$ T. Sain, J. Meaud, G. Hulbert, E. M. Arruda, and A. M. Waas, "Simultaneously high stiffness and damping in a class of wavy layered composites," Composite Structures 101, 104-110 (2013). 
${ }^{9}$ M. S. Chalhoub and J. M. Kelly, "Effect of bulk compressibility on the stiffness of cylindrical base isolation bearings," International Journal of Solids and Structures 26, 743-760 (1990).

${ }^{10}$ J. Weisman and G. P. Warn, "Stability of elastomeric and lead-rubber seismic isolation bearings," Journal of Structural Engineering 138, 215-223 (2011).

${ }^{11}$ M. Constantinou, A. Kartoum, and J. Kelly, "Analysis of compression of hollow circular elastomeric bearings," Engineering Structures 14, 103-111 (1992).

${ }^{12}$ G. Murray, F. Gandhi, and E. Hayden, "Polymer-filled honeycombs to achieve a structural material with appreciable damping," Journal of Intelligent Material Systems and Structures 23, 703-718 (2012).

${ }^{13}$ I. C. Finegan and R. F. Gibson, "Recent research on enhancement of damping in polymer composites," Composite Structures 44, 89-98 (1999).

${ }^{14}$ N. Ni, Y. Wen, D. He, X. Yi, T. Zhang, and Y. Xu, "High damping and high stiffness cfrp composites with aramid non-woven fabric interlayers," Composites Science and Technology 117, 92-99 (2015).

${ }^{15}$ J. Meaud, T. Sain, G. M. Hulbert, and A. M. Waas, "Analysis and optimal design of layered composites with high stiffness and high damping," International Journal of Solids and Structures 50, 1342-1353 (2013).

${ }^{16}$ F. Duc, P.-E. Bourban, C. Plummer, and J.-A. Månson, "Damping of thermoset and thermoplastic flax fibre composites," Composites Part A: Applied Science and Manufacturing 64, 115-123 (2014).

${ }^{17}$ R. Adams and M. Maheri, "Damping in advanced polymer-matrix composites," Journal of Alloys and Compounds 355, 126-130 (2003).

${ }^{18} \mathrm{C}$. Remillat, "Damping mechanism of polymers filled with elastic particles," Mechanics of Materials 39, 525-537 (2007).

${ }^{19}$ X. Zhou, E. Shin, K. Wang, and C. Bakis, "Interfacial damping characteristics of carbon nanotube-based composites," Composites Science and Technology 64, 2425-2437 (2004).

${ }^{20}$ J. Suhr, N. Koratkar, P. Keblinski, and P. Ajayan, "Viscoelasticity in carbon nanotube composites," Nature materials 4, 134 (2005).

${ }^{21}$ R. K. Patel, B. Bhattacharya, and S. Basu, "A finite element based investigation on obtaining high material damping over a large frequency range in viscoelastic composites," Journal of sound and vibration 303, 753-766 (2007). 
${ }^{22}$ I. Emri and B. von Bernstorff, "Dissipative bulk and granular systems technology," (2016), patent EP2700839.

${ }^{23}$ A. Kralj, T. Prodan, and I. Emri, "An apparatus for measuring the effect of pressure on the time-dependent properties of polymers," Journal of Rheology 45, 929-943 (2001).

${ }^{24}$ I. Emri and T. Prodan, "A measuring system for bulk and shear characterization of polymers," Experimental mechanics 46, 429-439 (2006).

${ }^{25}$ W. G. Knauss, I. Emri, and H. Lu, Mechanics of Polymers: Viscoelasticity. In Springer Handbook of Experimental Solid Mechanics, W. N. Sharpe (Ed.) (Springer Verlag, 2008) pp. 49-96.

${ }^{26}$ R. Fillers and N. Tschoegl, "The effect of pressure on the mechanical properties of polymers," Transactions of the Society of Rheology 21, 51-100 (1977).

${ }^{27} \mathrm{~W}$. Moonan and N. Tschoegl, "Effect of pressure on the mechanical properties of polymers. 2.expansivity and compressibility measurements," Macromolecules 16, 55-59 (1983).

${ }^{28} \mathrm{~W}$. Moonan and N. Tschoegl, "Effect of pressure on the mechanical properties of polymers. 3.substitution of the glassy parameters for those of the occupied volume," International Journal of Polymeric Materials and Polymeric Biomaterials 10, 199-211 (1984).

${ }^{29}$ M. Bek, J. Gonzalez-Gutierrez, J. A. M. Lopez, D. Bregant, and I. Emri, "Apparatus for measuring friction inside granular materials granular friction analyzer," Powder Technology 288, $255-265$ (2016).

${ }^{30}$ E. Jeram and R. Striker, "US4011929 (A)-Dampening device using a silicone rubber," (1977), US Patent 4,011,929.

${ }^{31}$ J. Fricke, "US5775049 (A)-Method and apparatus for damping structural vibrations," (1999), US Patent 5,924,261.

${ }^{32}$ A. Garcia, "US2007012530 (A1)-Bearing damper having dispersed friction damping elements," (2007), US Patent App. 11/183,135.

33 "DE20318708 (U1)-Shock absorber is made from recycled rubber granules," (2004), DE Patent 20,318,708.

${ }^{34}$ J. Bourinet and D. Le Houedec, "A dynamic stiffness analysis of damped tubes filled with granular materials," Computers \& structures 73, 395-406 (1999).

${ }^{35}$ W.-J. Wang, X.-Z. Kong, and Z.-G. Zhu, "Friction and relative energy dissipation in sheared granular materials," Physical Review E 75, 041302 (2007).

${ }^{36}$ S. Pamukcu and S. Akbulut, "Thermoelastic enhancement of damping of sand using syn- 
thetic ground rubber," Journal of Geotechnical and Geoenvironmental Engineering 132, $501-510$ (2006).

${ }^{37}$ J. Park and D. Palumbo, "Damping of structural vibration using lightweight granular materials," Experimental mechanics 49, 697-705 (2009).

${ }^{38}$ B. Darabi and J. Rongong, "Polymeric particle dampers under steady-state vertical vibrations," Journal of Sound and Vibration 331, 3304-3316 (2012).

${ }^{39}$ B. Darabi, J. Rongong, and T. Zhang, "Viscoelastic granular dampers under lowamplitude vibration," Journal of Vibration and Control , 1-14 (2016).

${ }^{40}$ A. Papalou and S. F. Masri, "Response of impact dampers with granular materials under random excitation," Earthquake Engineering \& Structural Dynamics 25, 253-267 (1996).

${ }^{41}$ R. D. Friend and V. Kinra, "Particle impact damping," Journal of Sound and Vibration 233, 93-118 (2000).

${ }^{42}$ M. Saeki, "Impact damping with granular materials in a horizontally vibrating system," Journal of Sound and Vibration 251, 153-161 (2002).

${ }^{43} \mathrm{Z} . \mathrm{Xu}, \mathrm{M} . \mathrm{Y}$. Wang, and T. Chen, "Particle damping for passive vibration suppression: numerical modelling and experimental investigation," Journal of Sound and Vibration 279, 1097-1120 (2005).

${ }^{44}$ M. Sánchez, G. Rosenthal, and L. A. Pugnaloni, "Universal response of optimal granular damping devices," Journal of Sound and Vibration 331, 4389-4394 (2012).

${ }^{45}$ M. Heckel, A. Sack, J. E. Kollmer, and T. Pöschel, "Granular dampers for the reduction of vibrations of an oscillatory saw," Physica A: Statistical Mechanics and its Applications 391, 4442-4447 (2012).

46 "Thermoplastic Polyurethane Elastomers (TPU), Elastollanß Product Range," (2016).

${ }^{47} \mathrm{CMS}$ stands for Center for Experimental Mechanics Measuring System.

${ }^{48}$ N. Tschoegl, W. Knauss, and I. Emri, "The effect of temperature and pressure on the mechanical properties of thermo-and/or piezorheologically simple polymeric materials in thermodynamic equilibrium - A critical review," Mechanics of Time-Dependent Materials 6, 53-99 (2002).

${ }^{49}$ J. D. Ferry, Viscoelastic properties of polymers (John Wiley \& Sons, 1980).

${ }^{50} \mathrm{~F}$. Murnaghan, "The compressibility of media under extreme pressures," Proceedings of the National Academy of Sciences 30, 244-247 (1944).

${ }^{51}$ M. Gergesova, B. Zupancic, I. Saprunov, and I. Emri, "The closed form t-T-P shifting 
(CFS) algorithm," Journal of Rheology 55, 1 (2011).

${ }^{52}$ M. Gergesova, I. Saprunov, and I. Emri, "Closed-form solution for horizontal and vertical shiftings of viscoelastic material functions in frequency domain," Rheologica Acta 55, 351-364 (2016).

${ }^{53}$ I. Emri and M. Gergesova, Time-dependent behavior of solid polymers. In Rheology: encyclopedia of life support systems, C. Gallegos (Ed.) (Eolss Publishers Co. Ltd., 2010) pp. 247-330.

${ }^{54}$ I. Emri, B. von Bernstorff, R. Cvelbar, and A. Nikonov, "Re-examination of the approximate methods for interconversion between frequency- and time-dependent material functions," Journal of Non-Newtonian Fluid Mechanics 129, 75-84 (2005).

${ }^{55}$ F. R. Schwarzl, "On the interconversion between viscoelastic material functions," Pure and applied Chemistry 23, 219-234 (1970).

${ }^{56}$ L. C. E. Struik, "Physical aging in plastics and other glassy materials," Polymer Engineering \& Science 17, 165-173 (1977). 